0

\title{
Variation in neuronal activity state, axonal projection target, and position principally define the transcriptional identity of individual neocortical projection neurons
}

\author{
Maxime Chevé $e^{1,2}$, Johanna D. Robertson ${ }^{3}$, Gabrielle H. Cannon ${ }^{4}$ \\ Solange P. Brown ${ }^{2, *}$ and Loyal A. Goff $f^{2,4}$
}

\section{Affiliations:}

${ }^{1}$ Biochemistry, Cellular and Molecular Biology Graduate Program, Johns Hopkins University School of Medicine, Baltimore, Maryland, 21205, USA.

${ }^{2}$ Solomon H. Snyder Department of Neuroscience, Johns Hopkins University School of Medicine, Baltimore, Maryland, 21205, USA.

${ }^{3}$ Human Genetics Training Program, McKusick-Nathans Institute for Genetic Medicine, Johns Hopkins University School of Medicine, Baltimore, Maryland, 21205, USA.

${ }^{4}$ McKusick-Nathans Institute for Genetic Medicine, Johns Hopkins University School of Medicine, Baltimore, Maryland, 21205, USA.

\section{${ }^{*}$ Corresponding Authors: spbrown@jhmi.edu (SPB) and loyalgoff@jhmi.edu (LAG)}




\section{ABSTRACT}

33 Single-cell RNA sequencing technologies have generated the first catalogs of transcriptionally

34 defined neuronal subtypes of the brain. However, the biologically informative cellular

35 processes that contribute to neuronal subtype specification and transcriptional heterogeneity

36 remain unclear. By comparing the gene expression profiles of single layer 6 corticothalamic

37 neurons in somatosensory cortex, we show that transcriptional subtypes primarily reflect

38 axonal projection pattern, laminar position within the cortex, and neuronal activity state.

39 Pseudotemporal ordering of 1023 cellular responses to manipulation of sensory input

40 demonstrates that changes in expression of activity-induced genes both reinforced cell-type

41 identity and contributed to increased transcriptional heterogeneity within each cell type. This

42 is due to cell-type specific biases in the choice of transcriptional states following manipulation

43 of neuronal activity. These results reveal that axonal projection pattern, laminar position, and

44 activity state define significant axes of variation that contribute both to the transcriptional

45 identity of individual neurons and to the transcriptional heterogeneity within each neuronal

46 subtype. 


\section{$47 \quad$ INTRODUCTION}

Single-cell RNA sequencing (scRNA-seq) approaches have been harnessed to reveal

49 previously hidden levels of complexity in cell types and states within a given tissue ${ }^{1,2}$. Nowhere

50 is this more relevant than in the mammalian central nervous system, an organ system

51 dependent on a remarkable diversity of cell types and cell states for its function. The neocortex

52 contains a wide variety of neuronal cell types organized into the circuits that direct higher brain

53 functions such as perception, memory and cognition. Furthermore, cortical neurons are highly

54 dynamic, undergoing significant changes in gene expression during development and

55 throughout adulthood in response to activity and experience ${ }^{3-8}$. Recent studies have generated a

56 more comprehensive 'parts list' of discrete cell types within the neocortex than previously

57 available ${ }^{9-16}$. While such surveys yield insights into the diversity of cortical cell types, the sources

58 of transcriptional variation both within and across cell types remain poorly understood ${ }^{17-19}$.

59 We compared expression profiles of layer 6 corticothalamic neurons (L6CThNs), a

60 heterogeneous population of related cortical projection neurons defined in anatomical,

61 functional, and gene expression studies, making them ideally suited for investigating the

62 relationships between transcriptional subtypes and other cellular properties ${ }^{12,16,20-29}$. By

63 combining scRNA-seq with an enrichment strategy that preserved axonal target information, we

64 identified two transcriptionally distinct $L 6 C T h N$ subtypes that each demonstrates a reproducible

65 bias for long-range projection targets. The transcriptional profiles of L6CThNs also reflect their

66 laminar position within L6. These two L6CThN subtypes exhibit divergent signatures of neuronal

67 activity both in their baseline expression patterns as well as in their responses following

68 manipulation of sensory input. The subtype biases in the choice of response following sensory

69 manipulation increased transcriptional heterogeneity both within and between cell types and 
70 reinforced the transcriptional identities of the two L6CThN subtypes. These results demonstrate

71 that scRNA-seq resolves relationships between gene expression and features such as axonal

72 projection pattern, spatial organization, and cell state, and identifies the independent

73 contributions of multiple biological signals that together determine transcriptional

74 heterogeneity within and across neuronal populations.

RESULTS

77 Transcriptional profiling of layer 6 corticothalamic neurons reveals two subtypes which reflect

\section{8 axonal projection bias}

79 Studies of primary sensory cortex demonstrate that L6CThNs are heterogeneous ${ }^{20-29}$. In rat

80 barrel cortex, L6CThNs in upper layer 6 project to the ventral posterior medial nucleus (VPM) of

81 the thalamus, while L6CThNs in lower layer 6 project to both VPM and the posterior medial

82 nucleus $(\mathrm{POm})^{21,25}$. To distinguish between these two projection classes in mouse, we first

83 validated Cre recombinase expression as a reliable marker for L6CThNs in the barrel cortex of

84 Neurotensin receptor 1-Cre mice ${ }^{30-32}$ (Fig. 1a-e; Ntsr1-Cre, Gensat 220). Next, we showed that a

85 subset of Cre-expressing, VPM-projecting L6CThNs in lower layer 6 also projects to POm (Fig. 1f-

86 j).

87 To determine whether L6CThNs subclasses projecting to VPM only or to both VPM and

88 POm are distinguished by their gene expression profiles, we labeled the two subclasses in

89 adolescent mice, microdissected barrel cortex containing retrogradely labeled cells, dissociated

90 the tissue into a single-cell suspension, sorted the differentially labeled L6CThNs using

91 Fluorescence Assisted Cell Sorting (FACS; Supplementary Fig. 1a-b), and collected enriched

92 populations of each subclass for bulk RNA sequencing. We identified 69 genes with significant 
differential expression (DE) between the two sorted populations (Fig. 1k, Supplementary File 1,

94 Supplementary Table 1; Cuffdiff2; 10\% false discovery rate (FDR)). These 69 DE genes demonstrate that subclasses of L6CThNs distinguished by their long-range axonal projection patterns are differentiated by their gene expression profiles. subclasses and may have obscured underlying transcriptional subtypes that comprise each

99 projection class of $\mathrm{L} 6 \mathrm{CThN}$ s. To resolve transcriptionally defined subtypes of L6CThNs, we next

100 evaluated the gene expression landscape of single L6CThNs using an unbiased classification

101 approach. We first sorted individual, labeled L6CThNs (Supplementary Fig. 1c) and collected 96

102 VPM-only and 96 VPM/POm L6CThNs from each of two replicate mice, totaling 384 single

103 L6CThNs. Individual cell lysates were subjected to a modified Smart-Seq2 library preparation

104 and scRNA-seq analysis. In total, 346 single L6CThNs passed quality control filters and were used

105 for further analysis (Supplementary Fig. 1d-i; Supplementary File 2). We confirmed the fidelity of

106 our enrichment by assessing each cell for neuronal and non-neuronal markers (Supplementary

107 Fig. 1j).

To identify transcriptional subtypes of L6CThNs, we selected genes with the greatest

109 likelihood of contributing to differences across the ensemble of single L6CThNs by identifying

110 genes with high residuals to a mean-variance model fit independently in each of the two

111 replicates and selecting the intersection of these two sets (Supplementary Fig. 2a). These 261

112 genes with high dispersion were therefore depleted for genes associated with technical

113 variation between replicates ${ }^{33,34}$ (Supplementary Table 2). Weights on the first three significant

114 principal components across all cells using this gene set $^{35}$ (Supplementary Fig. 2b; permutation

115 parallel analysis; $p<0.001$ ) were used for a tSNE dimensionality reduction followed by k-means 
117 two distinct subtypes. Fitting the data to three subtypes $(k=3)$ or more did not significantly

118 improve the performance of the clustering (Supplementary Fig. 2c). We compared our

119 classification approach to several recently described single-cell clustering utilities ${ }^{36,37}$ and found

120 a high-degree of agreement (Supplementary Fig. 2d; SC3: 93.77\% agreement; CIDR: 90\%

121 agreement). These results indicate that independent, unbiased clustering approaches based on

122 genes with higher than expected variance across the L6CThN population identify two major

123 subtypes of L6CThN.

$124 \quad$ To determine the relationship between transcriptional identity and morphological

125 subtypes, we next compared the distribution of VPM-only and VPM/POm projection labels

126 across the two transcriptionally defined subtypes (Fig. 2b). The majority of neurons in subtype 2

127 were labeled VPM-only (79\%, 103 of 130 cells, $\mathrm{p}<3.768 \times 10^{-17}$, hypergeometric test) while most

128 neurons in subtype 1 were VPM/POm (65\%, 141 of 216 cells, $p<3.768 \times 10^{-17}$, hypergeometric

129 test), a distribution significantly different from that expected by chance. Furthermore, the

130 axonal projection bias was reproducible across replicates (Fig. 2c) even though the segregation

131 of projection-defined subclasses was incomplete for both transcriptionally defined subtypes.

132 Together our results indicate that each transcriptionally defined subtype of L6CThN is enriched

133 for neurons targeting specific sets of thalamic nuclei.

\section{Transcriptional differences between subtypes of layer 6 corticothalamic neurons}

136 To assess the transcriptional differences between the two identified L6CThN subtypes, all

137 expressed genes were subjected to the Monocle2 differential test, using model formulas that

138 accounted for both batch effects and the number of genes detected in each cell, a proxy 
measure for efficiency of RNA capture and library synthesis. We identified 286 genes that were

140 significantly differentially expressed between the two subtypes (Fig. 2d; Monocle likelihood

141 ratio test, 0.1\% FDR; Supplementary File 2, Supplementary Table 3), only six of which overlapped

142 with the 69 DE genes observed in our bulk RNA-seq analysis of sorted L6CThNs despite the high

143 correlation between our bulk RNA-seq and scRNA-seq data (Supplementary Fig. 2e-g). This

144 result, in conjunction with our previous observation of incomplete label segregation across

145 L6CThN cell types, suggests that this discrepancy is primarily due to significant sample

146 heterogeneity arising from retrograde label inefficiencies. Importantly, parameters such as total

147 mapped fragments (mass), total estimated mRNAs per cell, number of genes detectably

148 expressed per cell, and replicate did not result in biased clustering across the DE gene list (Fig.

$1492 \mathrm{~d}$ ), suggesting a minimal influence of technical variation on the list of DE genes. The significant

150 differential expression of genes, including Fxyd6 and Lamp5, between the two subtypes was

151 consistent with expression patterns seen in the Allen Mouse Brain Atlas ${ }^{38}$ (Fig. 2e;

152 http://mouse.brain-map.org, Fxyd6-RP_051017_01_E10-coronal, Lamp5-RP_050725_01_B03-

153 coronal). The two subtypes also shared some transcriptional similarities with two recently

154 defined subtypes in primary visual cortex ${ }^{12}$ (Supplementary Fig. 3). Several long non-coding

155 RNAs (IncRNAs) were also specifically enriched in each subtype. For example, linc-Tmem $20^{8}$ was

156 significantly enriched in subtype 1 L6CThNs (Fig. 2f) and was preferentially expressed in lower

157 layer 6 (Fig. 2g). Conversely, the IncRNA Pantr1 was the gene with the greatest predictive power

158 for neurons in subtype 2 (Fig. $2 f, A U C=0.876$, power $=0.752, R O C$ analysis). Using a mouse line

159 in which LacZ was knocked into the Pantr1 locus ${ }^{39}$, we confirmed greater LacZ expression in

160 VPM-only L6CThNs relative to VPM/POm L6CThNs (Fig. 2h,i). Furthermore, in contrast to our

161 analysis of L6CThNs by anatomical labeling, we found that the total number of genes with 
162 detectable expression in each transcriptional subtype was significantly greater for subtype 1

163 relative to subtype 2, a finding consistent across replicates (Fig. $2 \mathrm{j}$ ), and that the mean pairwise

164 Euclidean distance between cells within each subtype was also greater in subtype 2 than in

165 subtype 1, indicating greater cell-cell variation across subtype 2 (Fig. $2 \mathrm{k}$; subtype $1 \mu=72.16$,

$166 \sigma=5.80$; subtype $2 \mu=80.59, \sigma=7.07 ; p<2.2 \times 10^{-16}$, Welch's two-sample t-test). Importantly, we

167 found only two genes, Lypd1 and Calm2, with a significant combinatorial effect of subtype and

168 label, suggesting that label does not distinguish subpopulations within each subtype. Together,

169 these data identify two L6CThN subtypes and confirm a relationship between transcriptionally

170 defined neuronal subtypes and the projection targets of these neurons.

171 To identify cellular processes that differentiate the two transcriptional subtypes of

172 L6CThNs, we queried the DE gene list for enrichment of annotated gene sets from public

173 databases (Supplementary Fig. 4a-d). Significant gene sets included Gene Ontology and

174 Reactome terms related to general features of neurons such as "Neuronal part" and "Synaptic

175 transmission" ( $p<0.01$; hypergeometric test, Benjamini-Hochberg corrected), highlighting the

176 limited resolution of gene sets in currently available public databases for generating biological

177 insights among neuronal subtypes. To identify more informative biological processes that shape

178 differences in the response properties of L6CThNs, we compared the expression of voltage-

179 gated ion channels, neurotransmitter receptors and neuropeptides, a number of which were

180 differentially expressed (Supplementary Fig. 4e-h). Several G protein-coupled receptors and

181 neuropeptides were differentially expressed. For example, Adcyap1 (PACAP) and a gene

182 encoding a peptide for processing PACAP, Pam, were preferentially expressed in subtype 2

183 (Supplementary Fig. 4h-i). Interestingly, receptors for PACAP are found in primary sensory

184 thalamic nuclei ${ }^{40}$ and modulate thalamocortical interactions ${ }^{41}$, consistent with the projection 
pattern bias of subtype 2 L6CThNs. Our data indicate that a focused analysis identifies gene expression differences that reflect relevant functional features.

\section{Distinct cellular processes are coordinately regulated within subtypes of L6CThN}

189 To identify cellular processes that contribute to the heterogeneity of gene expression across the 190 transcriptomes of all L6CThNs analyzed, we performed a weighted gene co-expression network 191 analysis (WGCNA) on all genes expressed in the $346 \mathrm{~L} \mathrm{CThNs}{ }^{42}$. This analysis yielded 22 modules 192 of co-regulated genes, which we classified using hierarchical clustering (Fig. 3a, Supplementary 193 Fig. 5a). We tested for significant correlations between subtype identity for each cell and the 194 eigenvalues of each module. Three modules significantly correlated with subtype 1 (Black, 195 Turquoise, and Cyan; Fig. 3b, Supplementary Fig. 5b; $p<0.01$; Pearson's product moment 196 correlation test) and four with subtype 2 (Red, Purple, Blue and Midnight Blue; Fig. 3b,

197 Supplementary Fig. 5b; $p<0.01$; Pearson's product moment correlation test). When the same 198 analysis was performed based on cell label, VPM-only or VPM/POm, correlation coefficients and 199 confidence measures were all weakened (Fig. 3b, Supplementary Fig. 5c). These seven modules 200 also showed significant correlation with the first principal component of the PCA on the high201 variance gene set (Fig. 3c, Supplementary Fig. 3h), suggesting that subtype identity explains a 202 significant amount of variation in gene expression across these neurons. Five of these modules 203 were enriched for genes identified as significantly differentially expressed between subtype 1

204 and subtype 2 (Fig. 3d, Supplementary Fig. 3d). No module was correlated with replicate or 205 other potentially confounding technical parameters (Supplementary Fig. 5f,g), confirming that 206 the variations we observe are driven by biological differences among neurons rather than 207 technical variation. Taken together, these data demonstrate that the greatest source of 
208 variation in the transcriptomes of L6CThNs is the difference between subtypes and reveal

209 several discrete modules of gene expression contributing to this difference.

211 Projection-dependent and position-dependent gene expression differences contribute to the

\section{2 transcriptional identity of layer 6 neurons}

213 Although the transcriptional signature of $\mathrm{L} 6 \mathrm{CThNs}$ reflects projection target bias, axonal

214 projection pattern and sublaminar position are confounded among L6CThNs. VPM/POm

215 neurons are predominantly located in lower layer 6 while VPM-only neurons are biased towards

216 upper layer 6 (Fig. 1h). Thus the gene expression differences we identified may represent

217 differences in sublaminar position within layer 6 rather than axonal projection pattern per se. To

218 test this hypothesis, we took advantage of the fact that L6CThNs represent only approximately

219 half of the neurons in layer $6^{29,31}$. If a gene's expression reflects axonal projection pattern, its

220 expression should be restricted to L6CThNs in upper or lower layer 6 only. On the other hand, if

221 a gene's expression reflects laminar position, we predict that its expression would be restricted

222 to neurons in either upper or lower layer 6, regardless of their projection pattern.

223 To select target genes to evaluate using single molecule fluorescence in situ

224 hybridization (smFISH), we performed a PCA on the mean-centered expression estimates of the

225 high-variance genes across all $346 \mathrm{~L} 6 \mathrm{CThNs}$ and used the rotations from this analysis to project

226 all expressed genes into this PCA space to rank order candidate genes (Supplementary Fig. 6a-d).

227 We quantified the expression of target gene mRNAs in individual tdTomato-positive, NeuN-

228 positive neurons identified as L6CThNs and in tdTomato-negative, NeuN-positive neurons in

229 slices of barrel cortex from Ntsr1-Cre;tdTomato mice. These data were then fit to a generalized

230 additive model to test the independent contributions of laminar position and expression of 
231 tdTomato (Fig. 4). We found that Lamp5, Serpini1 and Gabra5 were selectively expressed in a

232 subtype of L6CThNs (Fig. 4b,e-f). In contrast, Pantr1 varied with laminar position within layer 6

233 in both L6CThNs and non-L6CThNs (Fig. 4c). Our findings reveal that information about position

234 within layer 6 and long range axonal projection pattern is contained in the gene expression

235 differences between the two transcriptional subtypes of L6CThNs.

238 activity is known to strongly influence gene expression ${ }^{4-6}$. We therefore hypothesized that

239 neuronal activity state influences the transcriptional profiles of the L6CThNs and may contribute

240 to their transcriptional identity. To test whether any modules reflect activity state, we assessed

241 genes assigned to each module for enrichment of a curated gene set representing the ensemble

242 of genes regulated after induction of neural activity compiled from recently published studies ${ }^{43-}$

$243{ }^{46}$ (Fig. 3e, Supplementary Fig. 5, Supplementary Table 4). Four of the seven modules correlated

244 with transcriptional subtype (Black, Green, Purple, and Midnight Blue) were significantly

245 enriched for genes induced by neuronal activity ${ }^{43-46}$ (Fig. 3e, Supplementary Fig. 5, p $<0.01$,

246 hypergeometric test). Each of these four modules was also significantly correlated with the first

247 and second principal components of the PCA on the high-variance gene set, suggesting that

248 differences in neuronal activity state explain a significant amount of transcriptional variation

249 across $\mathrm{L} 6 \mathrm{CThNs}$ as well. Taken together, our results show that the long range axonal projection

250 pattern, laminar position within layer 6 , and the activity state of each neuron are all reflected in

251 the transcriptional profiles of individual L6CThNs and are principal contributors to the identity of

252 the two L6CThN subtypes.

253 Among the four activity-associated modules (Black, Green, Purple, and Midnight Blue), 
254 Black was specifically correlated with subtype 1, and Purple and Midnight Blue with subtype 2,

255 suggesting subtype-specific engagement of activity-induced genes in the steady state. The

256 Green module was correlated with PC1-PC3, but demonstrated no significant bias for either cell

257 type. To assess whether these signatures of neuronal activity represent a fundamental aspect of

258 subtype identity, we re-evaluated our classification workflow after regressing out the

259 eigenvalues for each activity-associated module and observed a reduction in the separation of

260 the two L6CThN subtypes in each case, suggesting that steady state differences in neuronal

261 activity genes are a defining characteristic of these two L6CThN subtypes (Supplementary Fig.

$2626 e)$.

To further test the influence of neuronal activity on transcriptional identity, we

264 evaluated the enrichment of activity genes along the first two principal components of a gene-

265 centric PCA of the mean-centered expression estimates of high variance genes across all 346

266 L6CThNs. All expressed genes were projected in this PCA space and ranked using their weights

267 along PC1 and PC2 (Supplementary Fig. 6a-d). Both PC1 and PC2 were significantly enriched for

268 genes drawn from our curated list of activity-induced genes ${ }^{43-46}(p<0.01$; Kolmogorov-Smirnov

269 test, Pre-ranked GSEA). The expression levels of the most heavily weighted PC1 genes varied

270 predominantly between subtypes (Fig. 5b-c), while the expression levels of heavily weighted PC2

271 genes additionally varied within each subtype in a pattern similar to classical activity-induced

272 genes like Fos and Bdnf (Fig. 5d-g), suggesting that activity contributes both to subtype identity

273 and to transcriptional heterogeneity within each subtype. The expression patterns of genes such

274 as Cdh13, Igfbp6, and Sla that strongly contributed to both PC1 and PC2 provide further

275 evidence that axonal projection pattern, laminar location, and activity state are not fully

276 orthogonal axes contributing to cellular identity (orange asterisks, Fig. 5c, Supplementary Fig. 
$2776 c, d)$. These data indicate that neuronal activity accounts for significant variation in the gene

278 expression of L6CThNs, contributing to transcriptional heterogeneity both within and between

279 L6CThN subtypes.

281 Modulation of neuronal activity influences the transcriptional state and identity of L6CThNs

282 Our data demonstrate that the gene expression profiles of L6CThNs reflect an integration of

283 multiple basis vectors corresponding to discrete, but potentially dependent sources of variation

284 dominated by axonal projection pattern, sublaminar position within layer 6 and neuronal

285 activity. Alterations in the molecular cascades engaged by different patterns of neural activity

286 therefore have the potential to modulate the transcriptional identity of L6CThNs. To test this

287 hypothesis, we unilaterally removed whiskers in a chessboard pattern, a pattern of sensory

288 deprivation shown to engage plasticity mechanisms in the barrel cortex ${ }^{47,48}$, from Ntsr1-

289 Cre;tdTomato mice which were bilaterally injected with a retrograde tracer in POm to label

290 VPM-only and VPM/POm L6CThNs as in our baseline experiments (Fig. 6a). At one and seven

291 days following this sensory manipulation, we collected and sequenced single L6CThNs from the

292 barrel cortex both contralateral and ipsilateral to the manipulation. After preprocessing and

293 quality control, we obtained high-quality transcriptional profiles for 133 of 151 sequenced

294 L6CThNs from 2 replicates one day following whisker removal (Day 1) and for 550 of 563

295 sequenced L6CThNs from 2 replicates seven days following whisker removal (Day 7). When

296 combined with L6CThNs collected under baseline conditions (Day 0), we generated a data set

297 comprised of 1023 sequenced L6CThNs collected from two replicates at each of three time

298 points (Supplementary Fig. 7a-d, Supplementary File 3).

299 To assign each newly acquired L6CThN to its transcriptional subtype, gene expression 
profiles were first pre-processed and normalized as described for the baseline data set (Day 0).

301 We then performed a PCA using the 286 genes we identified as differentially expressed between

302 transcriptional L6CThN subtypes under baseline conditions and a tSNE analysis on the 1023

L6CThNs (Fig. 6b). Neurons were clustered using a Gaussian mixture model ${ }^{49}, 50$ (MClust), and

304 cluster assignments were used to designate a subtype identity to each newly profiled neuron.

305 This approach largely recapitulated our original subtype assignments, as only ten neurons

306 classified under baseline conditions were assigned to a different subtype (Fig. 6c). The significant

307 axonal projection bias of the two transcriptional subtypes was maintained as subtype 1 neurons

308 were predominantly labeled VPM/POm (70.7\%, 408 of 585 cells, $p<2.97 \times 10^{-71}$, hypergeometric

309 test) and subtype 2 VPM-only (84.2\%, 369 of 438 cells, $p<2.97 \times 10^{-71}$, hypergeometric test),

310 highlighting the reproducibility of our method and the robustness of our subtype assignment

311 (Fig. 6d).

We identified 1134 genes with significant differential expression across the three time

313 points sampled, independent of stable, baseline differences in expression between the two

314 transcriptional subtypes (Fig. $6 \mathrm{e}, \mathrm{q} \leq 0.001$; Monocle2 test). A k-means clustering analysis of

315 mean expression profiles identified 16 clusters of genes with different temporal expression

316 following deprivation, but failed to identify any clusters with significant subtype-specific effects

317 over time (Fig. 6e). However, the transcriptional changes induced in response to altered sensory

318 input are unlikely to be synchronous across all neurons collected at each time point. To describe

319 the cellular responses to altered sensory input without the confounding effects of neurons in

320 diverse states intermixed at each time point, we established a pseudotemporal ordering for the

3211023 L6CThNs derived from the 1134 genes with significant differential expression across time

322 points (Fig. 7a). Briefly, using the Monocle2 DDRTree algorithm, cells were arranged in an 
323 embedded graph representation in a reduced dimensional space. In this manner, cells with

324 similar expression profiles across the 1134 target genes were positioned next to each other, and

325 a traversal through the graph revealed the sequence of transcriptional changes that reflect

326 progression through a given biological process. Through this projection, we captured a smooth

327 representation of transcriptional modulation to altered sensory input. As expected, the

328 distribution of L6CThNs along pseudotime generally followed the temporal order of collection

329 following whisker removal (Fig. 7a,b). Nonetheless, neurons from each time point were found

330 throughout pseudotime, confirming that the transcriptional response to sensory manipulation is

331 asynchronous across the population of L6CThNs. Interestingly, we observed no bias in the

332 distribution of L6CThNs across pseudotime when the neurons were grouped by hemisphere ipsi-

333 or contralateral to whisker removal, suggesting that longer term transcriptional responses in

334 L6CThNs from both hemispheres are similar (Supplementary Fig. 7e).

We identified 1507 genes that were differentially expressed across pseudotime at a

much higher stringency than our aggregate analysis independent of transcriptional subtype

337 designation ( $q \leq 0.0000001$; Monocle2), indicating that a significantly greater fraction of the

338 transcriptome was identified as modulated by sensory manipulation using the increased

339 resolution of pseudotemporal ordering. To identify cellular processes modulated along

340 pseudotime, we clustered the normalized response curves of the differentially expressed genes

341 (Fig. 7c, Supplementary Table 5) and queried each cluster for enrichment of annotated gene sets

342 from public databases and our curated list of activity-induced genes. The cluster with the

343 earliest changes in expression (Cluster 2) corresponded to significant downregulation of the

344 activity-associated genes ( $p<5.65 \times 10^{-7}$, hypergeometric test) that were enriched in the four

345 activity-associated modules contributing to cellular identity. In contrast, the cluster representing 
346 the late response (Cluster 3) corresponded to upregulation of genes associated with chromatin

347 remodeling and reorganization as well as upregulation of IncRNAs, suggesting a slower

348 epigenetic response to sensory manipulation. Interestingly, genes associated with long-term

349 potentiation (LTP) from Cluster 2 (Calm1, Calm2, Gria1, Gria2, Plcb1, Plcb4, Ppp1ca, and Ppp3r1)

350 were downregulated early in the response while LTP-associated genes in Cluster 3 (Crebbp,

351 Adcy1, Grin1, Prkcb, Ppp3ca, and $P p p 3 c b$ ) were upregulated towards the end of pseudotime,

352 suggesting that non-overlapping subsets of genes in this single gene set are regulated at distinct

353 phases of the L6CThN response to sensory manipulation. We also identified 75 transcription

354 factors (TFs) with significant differential regulation after sensory manipulation including activity-

355 associated $\mathrm{TFs}^{5,6}$ such as Arc, Fos, ler2, Junb, Mef2c, and Nr4a1, which were expressed early and

356 downregulated over the course of pseudotime. Several TFs involved in neural development ${ }^{3}$

357 including Neurod6, Fezf2, Mef2c, and Foxp2 were transiently expressed during the response,

358 suggesting a regulatory relationship between activity-dependent plasticity and neural

359 development.

Activity induced changes in gene expression enhance the distinction between subtypes

362 Our data demonstrate that a transcriptional signature of activity significantly contributes to

363 L6CThN subtype identity under baseline conditions, and that altered sensory inputs result in

364 dramatic transcriptional responses in L6CThNs. These results suggest that the response to

365 sensory manipulation may alter the transcriptional relationship among neurons of the same

366 subtype as well as the distinction between the two transcriptionally defined L6CThN subtypes.

367 To test these hypotheses, we assessed the distribution of pairwise Euclidean distances of the

368 variance stabilized gene expression estimates across all expressed genes for L6CThNs within 
each subtype for each day following sensory manipulation. In both subtypes, we observed that

370 the response to altered sensory input resulted in an increased mean distance among cells across

371 days (Fig. 8a; $p<2.2 \times 10^{-16}$, Welch's two-sample t-test). We also identified a significant increase

372 in the variance of the intra-subtype distances across days $\left(p<3.59 \times 10^{-5}\right.$, F-test $)$ for all adjacent

373 time points except for subtype 2 Day 1 versus Day 7, indicating that the cell-to-cell variation

374 within both L6CThN subtypes increases in response to altered sensory input. Second, we

375 assessed the distribution of the inter-subtype pairwise Euclidean distances between subtype 1

376 and subtype 2 . We found that the mean distance significantly increased from Day 0 to Day 1 as

377 well as from Day 1 to Day 7 (Fig. 8 b; $p<2.2 \times 10^{-16}$, Welch's two-sample t-test) as did the

378 variance of inter-subtype distances $\left(p<4.67 \times 10^{-8}\right.$, F-test $)$. These results demonstrate that

379 modulation of neuronal activity increases both the transcriptional heterogeneity within each

380 L6CThN subtype and the relative transcriptional differences between L6CThN subtypes,

381 confirming a dependent relationship between activity level and transcriptional identity.

\section{Subtype-biased responses contribute to transcriptional heterogeneity and subtype identity}

384 These results raise the possibility that subtype-specific responses to sensory manipulation drive

385 the increase in transcriptional heterogeneity and enhance the distinction between L6CThN

386 subtypes. We found that subtype 1 and subtype 2 were differentially distributed along

387 pseudotime at each time point assessed following sensory manipulation (Fig. 8c). Furthermore,

388 pseudotemporal ordering identified a single major branch point in the transcriptional responses,

389 with each of the two subsequent branches exhibiting a significant bias for a specific L6CThN

390 subtype (Branch A and Branch B; Fig. 8d). Consistent with the proportion of the two subtypes in

391 the baseline data, neither subtype 1 nor subtype 2 was significantly enriched in the root state 
392 (grey arrow; Subtype 1: $p<0.0715$; Subtype 2: $p<0.91$, hypergeometric test), indicating that

393 both transcriptional subtypes share a similar early response to sensory manipulation. In

394 contrast, Branch A and Branch B exhibited significant subtype bias: Branch A (red arrow) was

395 biased for subtype 1 (VPM/POM; 80.4\%, 148/184 neurons; $p<7.03 \times 10^{-14}$, hypergeometric test)

396 and Branch B (blue arrow) for subtype 2 (VPM-only; 58.4\%, 202/346 neurons; $p<2.04 \times 10^{-13}$,

397 hypergeometric test). Similar subtype-specific biases were seen at a subsequent branch point

398 (Supplementary Fig. 8). These results indicate that although either cell type may engage the

399 discrete processes represented by each branch in response to sensory manipulation, the cellular

400 decisions to engage a particular response are biased with respect to L6CThN subtypes.

4021392 genes with significant branch-dependent differential expression (Supplementary Table 6; q

$403 \leq 0.0001$; Monocle2 BEAM test); 926 of these overlapped with the 1507 genes with pseudotime-

404 dependent expression suggesting that discrete cellular responses independently contribute to

405 the aggregate transcriptional response to altered sensory manipulation. The branch-dependent

406 genes were organized into seven distinct clusters using hierarchical clustering of the Monocle2

407 branched model fits (Fig. 8e). Hypergeometric testing demonstrated that neurons that progress

408 along Branch A (VPM/POM enriched) were enriched for genes associated with engagement of

409 the proteasome complex, a process involved in synaptic remodeling ${ }^{51}$, while Branch B cells

410 (VPM-only enriched) were significantly enriched for genes related to the organization of the

411 postsynaptic density as well as to the regulation of LTP. We identified 79 TFs expressed in a

412 branch-dependent manner, including 31 TFs not identified in our initial analysis of the aggregate

413 response (Fig. 8f), suggesting that much of the aggregate L6CThN response induced by altered

414 sensory inputs is confounded across these two alternative cellular responses. The remaining 27 
415 pseudotime-dependent TFs may regulate a uniform response independent of these two

416 subtype-biased responses. Additional genes regulated in a branch-specific manner included

417 thirty-two presynaptic genes (Fig. 8g). Furthermore, neurons committing to a Branch A response

418 demonstrated enhanced expression of GABA receptor subunits (Gabra5, Gabrb1 and Gabrb2)

419 while neurons along Branch B induced ionotropic glutamate receptors (Fig. 8h; Grin1, Grin2b,

420 Grik5, and Grin2a). Taken together, these subtype-specific biases in transcriptional responses

421 induced by sensory manipulation likely underlie the overall effect we observed on L6CThN

422 identity, enhancing the distinctions between subtype 1 and subtype 2 , and significantly

423 increasing both the inter- and intra-subtype heterogeneity of L6CThNs.

\section{DISCUSSION}

Using scRNA-seq, we identified two transcriptionally defined subtypes of L6CThNs, each

427 exhibiting significant bias for particular long-range axonal projection targets. Analysis of gene

428 expression differences among the profiled neurons determined that variation in axonal

429 projection pattern, laminar position within the cortex, and neuronal activity state all significantly

430 contribute to transcriptional identity, and that these properties are at least partially dependent.

431 Manipulating the activity states of L6CThNs by altering tactile input further showed that each

432 subtype was biased for particular cellular responses to sensory manipulation, revealing

433 functional differences among these neurons. These subtype-biased transcriptional responses

434 not only increased cell-to-cell transcriptional heterogeneity within each subtype, but also

435 enhanced the transcriptional differences between the two subtypes. Together, these data

436 identify the most significant influences on the transcriptional identity of individual cortical

437 projection neurons, and suggest that discrete cellular properties and responses affect the 
438 population-level variation and identity of neuronal subtypes.

$439 \quad$ Although it is possible that projection target and transcriptional identity are decoupled

440 for a minority of neurons, the incomplete segregation of retrograde label across subtypes

441 observed here likely represents mislabeling of a subset of L6CThNs, as expected due to the close

442 proximity of VPM and POm and the difficulty of retrogradely labeling all neurons projecting to

443 adjacent targets. Interestingly, the subtypes identified here share some transcriptional

444 similarities with two recently defined subtypes in primary visual cortex ${ }^{12}$ (Supplementary Fig. 3),

445 suggesting a conservation of corticothalamic neuron transcriptional identity and axonal

446 projection target bias across functional regions of the cortex ${ }^{20}$. Future studies will be required to

447 determine whether VPM-only and LGN-only projecting neurons as well as VPM/POM and

448 LGN/LP neurons are transcriptionally analogous, and to identify sources of cellular variation

449 across cortical areas. Although the population of L6CThNs we analyzed is comprised of two

450 subtypes, profiling a greater number of neurons or profiling at greater depth may reveal

451 additional rare subtypes of $\mathrm{L} 6 \mathrm{CThNs}$ or may show that subtle variations in axonal projection

452 pattern identified in some anatomical studies are not apparent in the expression profiles of

453 cortical neurons ${ }^{20,21}$.

$454 \quad$ Pseudotemporal ordering of the states induced in L6CThN transcriptomes by altered

455 sensory input assessed over seven days revealed that L6CThNs engage at least two molecularly

456 distinct responses in a subtype-biased, but not subtype-specific, manner. Although the distinct

457 transcriptional responses were dominated by neurons collected one and seven days following

458 whisker manipulation, neurons collected in the baseline state were found throughout

459 pseudotime, suggesting that individual cortical neurons may engage these plasticity responses in

460 the non-manipulated state. L6CThNs in the initial phase of cellular response to altered sensory 
461 input exhibited similar gene expression changes, indicating that both subtypes engaged a

462 common initial transcriptional response. These results are consistent with previous studies of

463 responses induced by neural activity measured over hours across brain regions and between

464 inhibitory and excitatory neurons which found that common early transcriptional responses lead

465 to cell-type specific late responses ${ }^{52,53}$.

466 The differential response to sensory manipulation resulted in an increase in the

467 transcriptional variation across L6CThNs within each subtype as well as a significant

468 enhancement of the distinction between the subtypes, demonstrating a non-dissociable

469 relationship between neuronal identity and neuronal activity. Because single L6CThNs have the

470 potential to engage either transcriptional response regardless of subtype, our data suggest that

471 extrinsic factors such as distinct activation patterns, due to differences in the circuits in which

472 each subtype is embedded, induce neurons from a given subtype preferentially toward a similar

473 response rather than solely cell-autonomous properties. Interestingly, we found that the

474 transcriptional responses for neurons ipsilateral and contralateral to the sensory manipulation

475 were similar, suggesting that shared cellular mechanisms underlie the previously described

476 functional effects ipsilateral and contralateral to unilateral manipulation at timescales similar to

477 those assessed here ${ }^{48,54,55}$. Furthermore, expression of genes that strongly contributed to

478 subtype identity such as $\operatorname{Lamp} 5$ was altered in response to sensory manipulation. These data

479 indicate that factors that change cell state such as plasticity or injury affect our ability to

480 accurately define discrete, stable transcriptional subtypes.

481 Our results indicate that the transcriptional profiles of cortical neurons reflect specific

482 features of these cells, but also that the transcriptional variation across individual neurons is a

483 principal feature of subtype identity with significant functional consequences for individual 
484 neuronal responses and subtype function. Subtype 2 L6CThNs were more transcriptionally

485 heterogeneous than subtype 1 neurons under steady-state conditions in part because of

486 baseline differences in gene expression associated with neural activity. In addition, the intra-cell

487 type variation across subtype 2 at day 1 and at day 7 was greater than the inter-cell type

488 variation at the same time points, suggesting that changes in cell-to-cell variation, rather than

489 subtype specific differences, dominate the transcriptional responses to experience dependent

490 plasticity. Together, these data indicate that the contribution of neuronal activity to gene

491 expression differs across distinct neuronal subtypes and that the transcriptional variation due to

492 differences in neuronal activity state plays a central role in defining the identity of cortical

493 projection neurons. 


\section{METHODS}

\section{Mice}

496 All procedures were approved by the Johns Hopkins Animal Care and Use Committee and 497 followed the guidelines of the Society for Neuroscience and the National Institutes of Health.

498 Animals used for RNA-sequencing ranged from postnatal day 23 (P23) to P28; animals used for

499 immunohistochemistry and in situ hybridization ranged from P23 to P32. Both males and females were used in this study (gender indicated for each replicate below). All animals were kept on a $12 \mathrm{~h}$ light/dark cycle, housed with at least two mice per cage and provided with unlimited water and food. The following mouse lines were used:

Neurotensin receptor-1 Cre recombinase line ${ }^{30}$ (Ntsr1-Cre, GENSAT 220), loxP-STOP-loxPtdTomato Cre reporter lines ${ }^{56}$ (Ai9 and Ai14, Allen Institute for Brain Science), loxP-STOP-loxPeYFP Cre reporter line ${ }^{56}$ (Ai3, Allen Institute for Brain Science), and Pantr1-LacZ ${ }^{39}$. Mice were maintained on mixed backgrounds including C57BL/6 and CD-1.

\section{Stereotactic injections}

To identify layer 6 corticothalamic neurons (L6CThNs), mice P18 to P23 were anesthetized with ketamine $(50 \mathrm{mg} / \mathrm{kg})$, dexmedetomidine $(25 \mu \mathrm{g} / \mathrm{kg})$ and the inhalation anesthetic, isoflurane. Animals were placed in a custom-built stereotactic frame and anesthesia was maintained with isoflurane (1-2.5\%). A small craniotomy was performed, and a glass pipette (10-25 $\mu \mathrm{m}$ tip diameter) was lowered into the thalamus. To identify L6CThNs that project to the ventral posterior medial nucleus (VPM) of the thalamus, Ntsr1-Cre;tdTomato mice were injected in VPM (1.1 $\mathrm{mm}$ posterior, $1.7 \mathrm{~mm}$ lateral , $1.4 \mathrm{~mm}$ ventral from bregma) with either Alexa 488 Cholera toxin B (CTB488, ThermoFisher) or green Retrobeads IX (Lumafluor), and Ntsr1-Cre;YFP mice were injected with Alexa 555 Cholera toxin B (CTB555, ThermoFisher). To identify L6CThNs that project to both VPM and to the posterior medial nucleus of the thalamus (POm), Ntsr1Cre;tdTomato mice were injected in POm (1.35 mm posterior, $1.23 \mathrm{~mm}$ lateral , $3.3 \mathrm{~mm}$ ventral from bregma) with green Retrobeads IX, and Ntsr1-Cre;YFP mice were injected with either CTB555 or red Retrobeads (Lumafluor). Between 30 and $100 \mathrm{nl}$ of tracer were pressure-injected, and the pipette was kept in position for 5-10 minutes before removal. Following the injection, the incision was sutured and buprenorphine $(0.05 \mathrm{mg} / \mathrm{kg})$ was administered to all animals postoperatively. Quantification of the colocalization between retrogradely labeled neurons and Cre expression in Ntsr1-Cre mice (Fig. 1e,j) was performed on mice injected with either CTB or Retrobeads. Preliminary experiments indicated that all tracers used generated similar labeling. However, Retrobeads resulted in increased discrimination of labeled neurons during Fluorescence Assisted Cell Sorting (FACS) and were therefore used for all FACS experiments.

\section{Immunohistochemistry}

Brains from animals injected with neuronal tracers were removed and placed in a solution of $4 \%$ paraformaldehyde (PFA) in $0.01 \mathrm{M}$ phosphate buffered saline (PBS) for $3 \mathrm{~h}$. Coronal sections were cut on a vibratome (50 $\mu \mathrm{m}$, VT-1000S, Leica). Mice expressing YFP were subjected to immunohistochemistry (1:1000, chicken anti-GFP, GFP-1020, Aves, RRID:AB_10000240, and 1:300 AlexaFluor 488-conjugated goat anti-chicken, Life Technologies, A11039, RRID:AB_10563770). Sections were then mounted using Aqua Poly/Mount mounting medium (Polysciences, Inc) and visualized on a confocal microscope (LSM 510, Zeiss) using 10x (0.3 NA), $25 x(0.8 \mathrm{NA}$ ) or 40x (1.3 NA) objectives or on an epifluorescence microscope (AxioObserver.Z1, 
Zeiss) using a $5 \times(0.16 \mathrm{NA})$ objective. Colocalization of tdTomato, YFP and retrograde tracer was quantified using single-plane confocal images and the Cell Counter plugin in Fiji ${ }^{57}$. For all anatomical comparisons, upper layer 6 and lower layer 6 were defined as the top $50 \%$ and bottom $50 \%$ of the layer defined by Cre-expressing neurons in Ntsr1-Cre mice.

\section{Cell isolation and enrichment}

For bulk sequencing experiments (bulk RNA-seq), POm was injected with green Retrobeads in three Ntsr1-cre;tdTomato mice. For single-cell sequencing experiments (scRNA-seq), POm was injected with red or green Retrobeads in Ntsr1-Cre;YFP mice or in Ntsr1-Cre;tdTomato mice, respectively. Mice were sacrificed 5-7 days after surgery (Bulk RNA-seq: Replicate 1: P23 female; Replicate 2: P23 female; Replicate 3: P26 male; scRNA-seq baseline: Replicate 1: P27 male, Replicate 2: P28 female; scRNA-seq Day 1: Replicate 1: P24 female, Replicate 2: P24 female; scRNA-seq Day 7: Replicate 1: P29 female, Replicate 2: P27 female). Brains were rapidly removed and $300 \mu \mathrm{m}$ thick somatosensory thalamocortical ${ }^{58}$ or coronal slices were sectioned (VT-1200s, Leica) in ice-cold sucrose solution containing the following (in $\mathrm{mM}$ ): $76 \mathrm{NaCl}, 25$ $\mathrm{NaHCO}_{3}, 25$ glucose, 75 sucrose, $2.5 \mathrm{KCl}, 1.25 \mathrm{NaH}_{2} \mathrm{PO}_{4}, 0.5 \mathrm{CaCl}_{2}, 7 \mathrm{MgSO}$, pH 7.3, $310 \mathrm{mOsm}$ continuously bubbled with $95 \% \mathrm{O}_{2} / 5 \% \mathrm{CO}_{2}$. The slices were then placed in a submersion chamber on an upright microscope in ice-cold sucrose solution continuously bubbled with $95 \% \mathrm{O}_{2} / 5 \% \mathrm{CO}_{2}$. The barrel cortex was identified using differential interference contrast (DIC) and epifluorescence microscopy (Axioskop2 FsPLus, Zeiss) using 4x (0.1 NA) and 40x (water immersion, $0.75 \mathrm{NA}$ ) objectives. The location of the retrograde tracer in the lower half of layer 6 was visually confirmed, and then regions of cortex below barrels with appropriate labeling were microdissected and immediately dropped into $2.6 \mathrm{~mL}$ equilibrated Papain DNase-I solution. For all experiments, at least three consecutive slices confirmed as clearly labeled were microdissected.

The dissociation protocol was adapted from the trehalose-enhanced neuronal isolation protocol described in Saxena et al, $2012^{59}$ which is based on the Worthington Papain Dissociation System (Worthington Biochemical Corporation). Briefly, tissue pieces were dropped into a $5 \mathrm{ml}$ tube containing $2.6 \mathrm{~mL}$ of a trehalose-Papain-DNase1 solution which included RNase inhibitor, incubated $30-45 \mathrm{~min}$ at $37^{\circ} \mathrm{C}$ with gentle trituration at the half way point using a p1000 pipetter. The reaction was stopped by adding a protease inhibitor solution (for solution compositions, see Saxena et al, $2012^{59}$. The tissue was then further dissociated and washed by sequential pipetting and low-speed centrifugations. The final pellet was resuspended in $200 \mu \mathrm{L}$ of media (DMEM, 5\% trehalose (w/v), $25 \mu \mathrm{M} \mathrm{AP-V}, 0.4 \mathrm{mM}$ kynurenic acid, $6 \mu \mathrm{L}$ of $40 \mathrm{U} / \mu \mathrm{l}$ RNase inhibitor) at room temperature and introduced into a FACS machine (Beckman Coulter MoFlo Cell Sorter). Neurons were sorted based on their fluorescence (either tdTomato-positive (red) and Retrobead-positive (green) versus tdTomato-positive only or YFP-positive (green) and Retrobead-positive (red) versus YFP-positive only) directly into lysis buffer either in eppendorf tubes for bulk sequencing (350 $\mu \mathrm{L}$ lysis buffer, Qiagen RNeasy Micro Kit; Replicate 1: tdTomatoonly: 1943 cells, tdTomato and tracer: 2287 cells; Replicate 2: tdTomato-only: 386, tdTomato and tracer: 782; Replicate 3: tdTomato-only: 604, tdTomato and tracer: 934) or into individual wells of 96-well plates for single-cell sequencing $(2 \mu \mathrm{L}$ Smart-Seq2 lysis buffer + RNAase inhibitor, $1 \mu \mathrm{L}$ oligo-dT primer, and $1 \mu \mathrm{L}$ dNTPs according to Picelli et al, 2014 ${ }^{60}$. Upon completion of a sort, the plates were briefly spun in a tabletop microcentrifuge and immediately placed on dry ice. Single-cell lysates were subsequently kept at $-80^{\circ} \mathrm{C}$ until cDNA conversion.

584 


\section{Library preparation and amplification}

For bulk samples, RNA was extracted with a Qiagen RNeasy Micro Kit. RNA quality was assessed with the Bioanalyzer Pico RNA kit and samples had RIN scores of 8.2-8.8. Library preparation and amplification were performed according to the Smart-Seq2 protocol $^{60}$ with some modifications. Principally, all primer and adapter sequences were synthesized with a 5'-biotin to minimize primer dimerization. Briefly, 200 pg of RNA per sample were used as input for template switching cDNA synthesis. Full-length cDNA was amplified by KAPA HiFi mediated PCR for 20 cycles using a 5'-biotinylated ISPCR primer. Then, 500 pg of Ampure XP bead cleaned (1:1) cDNA was used as input for a standard Nextera XT tagmentation reaction, and amplification of adapter-ligated fragments was carried out for 12 cycles during which individual index sequences were added to each distinct sample. Library concentration was assessed with Qubit and library fragment size distribution was assessed on the Agilent Bioanalyzer. Pooled, indexed bulk RNAseq samples were initially sequenced on the Illumina MiSeq 500 platform, and subsequently the same pool was sequenced on one lane of the Illumina HiSeq 2500 platform to produce $50 \mathrm{bp}$ paired-end reads. Libraries were sequenced to an average depth of $4.2 \times 10^{7}$ fragments each. Reads from both runs were aggregated by index prior to mapping.

Library preparation and amplification of single-cell samples was performed using a modified version of the Smart-Seq2 protocol. Briefly, 96-well plates of single-cell lysates were thawed to $4^{\circ} \mathrm{C}$, heated to $72^{\circ} \mathrm{C}$ for 3 minutes, and then immediately placed on ice. Template switching first-strand CDNA synthesis was performed as described above using a $5^{\prime}$-biotinylated TSO oligo. cDNAs were amplified using 18 cycles of KAPA HiFi PCR and 5'-biotinylated ISPCR primer. Amplified cDNA was cleaned with a 1:1 ratio of Ampure XP beads and $300 \mathrm{pg}$ was used for a one-half standard-sized Nextera XT tagmentation reaction. Tagmented fragments were amplified for 12 cycles and dual indexes were added to each well to uniquely label each library. Concentrations were assessed with Quant-iT PicoGreen dsDNA Reagent (Invitrogen) and samples were diluted to $\sim 2 \mathrm{nM}$ and pooled. Pooled libraries were sequenced on the lllumina HiSeq 2500 platform to a target mean depth of $\sim 8.0 \times 10^{5} 50$ bp paired end fragments per cell ${ }^{61}$ at the Hopkins Genetics Research Core Facility to generate 50 bp paired-end reads.

\section{In situ hybridization and immunohistochemistry}

Immunohistochemistry (IHC) for tdTomato and in situ hybridization (ISH) for linc-Tmem20 were combined to show co-localization of the IncRNA transcript and tdTomato protein in L6CThNs. Ntsr1-Cre;tdTomato mice (P23 to P30) were transcardially perfused with $0.1 \mathrm{M}$ PBS followed by a $4 \%$ PFA solution, and brains were post-fixed for $3 \mathrm{~h}$ at room temperature in $4 \%$ PFA. Then, 30 $\mu \mathrm{m}$ sections were cut on a vibratome (VT-1000S, Leica) and first processed for IHC (1:1000, Rabbit anti-DsRed polyclonal antibody, Living Colors, Clontech Laboratories, RRID: AB_10013483 and 1:300 AlexaFluor 568-conjugated goat anti-rabbit, Life Technologies, A11011, RRID: $\left.A B \_143157\right)$. Next, the sections were processed for ISH following the protocol described in Blackshaw, $2013^{62}$ without proteinase $\mathrm{K}$ treatment. Sections were incubated overnight at $65^{\circ} \mathrm{C}$ with $5 \mathrm{ng} / \mu \mathrm{L}$ of an anti-sense linc-Tmem 20 DIG labeled probe (DIG RNA labeling Kit (SP6/T7), Roche). Detection of the hybridized DIG probes was performed using an alkaline phosphatase (AP) conjugated antibody (1:1000, Anti-Digoxigenin [21H8] Alkaline Phosphatase, Abcam, Cat. No.: ab119345, RRID:AB_10901703) followed by application of the AP substrate BCIP/NBT (SigmaFast BCIP/NBT, Sigma-Aldrich). The enzymatic reaction was carried out until the desired signal to noise ratio was observed. Images were then taken on an AxioObserver.Z1 (Zeiss) using 
a combination of epifluorescence and brightfield imaging (10x objective, NA: 0.3). Brightness and contrast were adjusted using Adobe Photoshop (Adobe Systems Incorporated).

For validation of Pantr1 expression, transgenic mice expressing LacZ driven by the endogenous Pantr1 promoter locus ${ }^{39,63}$ were injected with CTB488 in VPM and CTB555 in POm. IHC was performed on $50 \mu \mathrm{m}$-thick sections (rabbit anti- $\beta$ galactosidase antibody 1:5000; a kind gift from Dr. Joshua R. Sanes, Harvard University, Cambridge, MA and 1:300 AlexaFluor 647conjugated donkey anti-rabbit, Life Technologies, A31573, RRID: AB_2536183). Imaging was performed on a confocal microscope (LSM 510, Zeiss) using 10x (0.3 NA), 25x (0.8 NA) or 40x (1.3 NA) objectives. LacZ-positive and retrogradely labeled neurons were manually counted on single-plane confocal images using the Cell Counter plugin in $\mathrm{Fiji}^{57}$. Brightness and contrast were adjusted using Adobe Photoshop (Adobe Systems Incorporated).

For detection of single mRNA molecules, we used the commercially available system RNAscope (ACDbio). Ntsr1-Cre;tdTomato mice (P23 to P30) were transcardially perfused with $0.1 \mathrm{M}$ PBS followed by a 4\% PFA solution, and brains were post-fixed for $2 \mathrm{~h}$ at room temperature in $4 \%$ PFA. Fixed brains were then washed in PBS and allowed to equilibrate in a $30 \%$ sucrose-PBS solution for $36 \mathrm{~h}$ before being embedded and frozen in OCT compound (Scigen, Tissue-Plus \#4583). Frozen brains were kept at $-80^{\circ} \mathrm{C}$ for up to 2 months. $20 \mu \mathrm{m}$ sections were collected on a cryostat (Leica $\mathrm{CM} 3050$ ) and processed according to instructions provided by RNAscope. The following probes were used: tdTomato-C2 (317041-C2), tdTomato-C3 ( 317041-C3), Mm-Rbfox3 (313311), Rbfox3-C2 (313311-C2), Mm-Lamp5 (451071), Mm-Pantr1 (483711), Mm-Serpini1 (501441), Gabra5-C3 (319481-C3). Imaging was performed on a confocal microscope (LSM 510, Zeiss) using a 63x objective (NA 1.4). Sections from three animals were used for Pantr1, Lamp5 and Gabra5 and from four animals for Serpini1. For quantification of the number of mRNAs per neuron obtained using RNAscope, single plane images were first processed with the open-source software CellProfiler (Broad Institute) to automatically identify individual cells based on DAPI signal and to generate outline files. These outlines were then used as input, in combination with the corresponding confocal images, into FISH-quant ${ }^{64}$ (Matlab) for quantification of transcript number per cell. Cells with no detectable NeuN (Rbfox3) transcript were discounted as non-neuronal cells. A threshold of 20 tdTomato puncta was selected as the cutoff for distinguishing tdTomato-positive from tdTomato-negative neurons. This resulted in 164 tdTomato-positive and 188 tdTomato-negative neurons for Serpini1, 118 tdTomato-positive and 150 tdTomato-negative neurons for Lamp5, 111 tdTomato-positive and 198 tdTomatonegative neurons for Pantr1, and 135 tdTomato-positive and 160 tdTomato-negative neurons for Gabra5. Individual images were stitched to reconstitute an image spanning layer 6 vertically, and the distance of each neuron from the bottom of the layer was measured manually using $\mathrm{Fiji}^{57}$ and normalized to the height of layer 6 . Statistical testing (Likelihood ratio test) and curve fitting (Loess) was performed in R/Bioconductor.

\section{Sensory manipulation}

Prior to sensory manipulation, animals were bilaterally injected in POm with green Retrobeads IX (Lumafluor) as described above. To induce experience dependent plasticity, mice (P18 to P23) were anesthetized with the inhalation anesthetic, isoflurane. Once an adequate level of anesthesia was achieved, the animals were placed under a surgical microscope and, using tweezers, individual vibrissae were gently pulled by applying slow, steady tension to the base of the whisker ${ }^{65}$. To produce a chessboard pattern, vibrissae alpha, gamma, A2, A4, B1, B3, C2, C4, C6, D1, D3, D5, D7, E1, E3, E5 and E7 were pulled unilaterally while the rest remained 
untouched. Following the procedure, the animals were returned to their home cage and housed with at least one other animal until tissue collection. We checked for regrowing vibrissae every two days and removed them if visible.

\section{Read pre-processing}

For both bulk and single-cell libraries, paired-end reads were aligned to the mouse reference genome (mm10) using Hisat $2^{66}$ with the default parameters except: $-p$. Aligned reads from individual samples were quantified against a reference transcriptome ${ }^{61}$ (GENCODE vM8) supplemented with additional IncRNA genes described previously ${ }^{8}$ (Supplementary File S1) with cuffquant ${ }^{67}$ using default parameters with the following exceptions: --no-update-check $-p$ 8. For bulk RNA-Seq, pre-processed expression estimates were used as input for cuffdiff using default parameters except: - $p 16$, and the output of cuffdiff was analyzed using the cummeRbund $\mathrm{R} /$ Bioconductor package ${ }^{68}$. For single-cell experiments, normalized expression estimates across all samples were obtained using cuffnorm with default parameters. For comparison to results from primary visual cortex ${ }^{12}$, raw reads were downloaded from the short read archive (GSE71585), processed as described above for single-cell samples, and normalized expression estimates were obtained from combining both sources of single-cell reads together via cuffnorm.

\section{Single-cell analysis}

The normalized FPKM matrix from cuffnorm was used as input for the Monocle2 single-cell RNAseq framework ${ }^{69}$ in R/Bioconductor ${ }^{70}$. Relative FPKM values for each cell were converted to estimates of absolute mRNA counts per cell (RPC) using the Monocle2 census utility. A total of 346 individual cells under baseline conditions passed quality filters including: a) total mRNAs between 2000 and 200,000 per cell, and b) >10,000 fragments mapped (total_mass). 12,537 genes were identified as expressed in at least 15 cells across all conditions.

To identify high-variance genes, a generalized additive model ${ }^{71}$ (MGCV R package) was fit to the $\log _{2}$ mean RPC expression versus a cubic spline fit to the $\log _{2}$ coefficient of variation(BCV) for each replicate dissociation independently (Supplementary Fig. 2a and Fig. 7d). The intersection of genes with residuals to this fit greater than 1.0 from each replicate were chosen as 'high-variance' genes and the $\log _{2}$ expression estimates (with a pseudocount of 1 ) of these selected genes were used as input for PCA analysis and t-stochastic nearest neighbor embedding (tSNE) clustering of individual cells ${ }^{72}$.

To cluster individual cells, we employed a spectral tSNE approach ${ }^{73}$, combining nonlinear dimensional reduction and density clustering. Briefly, PCA analysis was conducted on high-variance genes and then a subset of components $(n=3$, selected by permutation parallel analysis, Supplementary Fig. 2 b) were used as input for the tSNE visualization algorithm ${ }^{72}$ (perplexity = 30). K-means clustering was used to partition cells using the two reduced tSNE dimensions into two discrete subtypes. The choice of $k$ was made using 1000 bootstrapped tSNE estimates and evaluating $\mathrm{k}=2$ through $\mathrm{k}=8$ for each round. The average silhouette coefficient for each $\mathrm{tSNE}^{*} \mathrm{k}$ was determined and the mean and standard deviation of these estimates was estimated for each $\mathrm{k}$ (Supplementary Fig. $2 \mathrm{c}$ ). $\mathrm{k}=2$ was chosen as the $\mathrm{k}$ value with the highest average silhouette coefficient and the lowest value of $k$ for which there was no significant improvement in mean silhouette score.

After cluster assignment, differential expression testing was performed across all expressed genes using the Monocle2 VGAM model comparison test ${ }^{69}$. To test for differentially 
expressed genes between transcriptionally-defined cell types, the following full model was fit to each expressed gene: replicate+num_genes_expressed+celltype, and compared to a reduced model in which celltype was removed. The number of genes expressed in each cell was included as an explanatory variable as a proxy for the sensitivity of scRNA-seq in each individual cell. To test for differentially expressed genes between anatomically labeled cells, the following full model was fit to each expressed gene: replicate+num_genes_expressed+label, and compared to a reduced model in which label was removed. In both cases, genes were selected as significantly differentially expressed with a $0.1 \%$ false discovery rate (Monocle 2 test; BenjaminiHochberg corrected). DE gene lists were tested for gene set enrichment against Gene Ontology and Reactome genesets ${ }^{74,75}$ using the clusterProfiler Bioconductor package ${ }^{76}$. The heatmap of differentially expressed genes was composed using the pheatmap R package ${ }^{77}$ and dendrograms were generated from the hierarchical clustering of the Euclidean distances between points.

To identify gene sets contributing to transcriptional identity in our single-cell dataset, gene-centric PCA was performed on a mean-centered matrix of variance-stabilized expression estimates for high-variance genes across all cells. The resulting rotations were used to project all expressed genes into the same PCA space to identify their weights. These weights were used to rank-order all expressed genes and this ordering was used as input for a pre-ranked GSEA analysis $^{78}$. Gene sets for the GSEA analysis were derived from the Monocle2 differential gene tests described above or from our curated list of neuronal activity genes ${ }^{43-46}$ using their adjusted $p$-value cutoff of $p<0.01$ (Supplementary Table 4).

\section{WGCNA}

To identify modules of correlated gene expression we used the Weighted Gene Correlation Network Analysis package WGCNA ${ }^{42}$. Normalized expression data were first batch-corrected with respect to replicates using limma, and the resulting matrix of 12,537 detectably expressed genes and 346 cells was used as input for WGCNA. Cells were hierarchically clustered using the Jensen-Shannon distance with average linkage. A soft threshold power of 11 was used to create a signed Topological overlap matrix directly from the normalized and batch-corrected expression estimates. Modules were then learned with a minimum module size of 30 . Modules were merged below a distance threshold of 0.5 . Module eigenvalues were estimated and correlated with cellular traits using the Pearson product moment correlation test. Module gene membership was determined in a similar manner in which individual gene expression estimates were correlated with module eigenvalues and tested for significance. To test the effect of each module on the segregation of L6CThN cell types, each module eigenvalue was separately regressed out of the expression matrix using limma, and subsequent values were used as input for a tSNE using identical parameters to the original assay.

\section{Pseudotime and BEAM analyses}

Pseudotemporal ordering was performed using the prescribed Monocle2 workflow. Briefly, all $1023 \mathrm{~L} 6 \mathrm{CThN}$ s passing quality controls were used as input. To reconstruct a trajectory that reflected cellular progression in response to altered sensory input, we first performed a differential test to identify genes whose expression changed as a function of collection day, independent of baseline differences between celltype (fullModelFormulaStr = " num_genes_expressed+Total_mRNAs+sex+celltype*Day, reducedModelFormulaStr = " num_genes_expressed+Total_mRNAs+sex+celltype", q $\leq 0.01$ ). These 1134 genes were used as 
a filtering set for the DDRTree dimensionality reduction. Cells were tested for differential expression along pseudotime using the following model comparisons with $q \leq 0.0000001$ : fullModelFormulaStr = " num_genes_expressed+celltype+sm.ns(Pseudotime,df=3)", reducedModelFormulaStr $=$ " num_genes_expressed+celltype". Branch dependent expression was determined using the Monocle2 BEAM test (fullModelFormulaStr $=$ " num_genes_expressed+celltype+sm.ns(Pseudotime, $d f=$ 3)*Branch",reducedModelFormulaStr = " num_genes_expressed+celltype+sm.ns(Pseudotime, $d f=3) ", q \leq 0.0001$ ). All significant gene lists were tested for gene set enrichment using the hypergeometric test.

\section{Data and software availability}

All primary data are archived on the SRA and Gene Expression Omnibus and are available for direct download on our companion website (http://rstudio.gofflab.org:8383/apps/L6CthPN_dash/). Source code and software tools are available upon request.

Author Contributions: MC, SPB and LAG conceived the experiments. MC, JDR, and GHC performed all experiments. MC, SPB and LAG analyzed the data and wrote the paper with input from all authors.

Conflict of Interest: The authors declare no competing financial interests.

Acknowledgments: We thank Hao Zhang and the Johns Hopkins Bloomberg School of Public Health Flow Cytometry and Cell Sorting Core Facility. Sequencing service was provided by the Johns Hopkins Genetic Resources Core Facility. We thank Dr. Joshua R. Sanes, Harvard University, for the anti-LacZ antibody and Dr. Jesse Gray, Harvard Medical School, for the curated list of neuronal activity-induced genes. This work was supported by a KlingensteinSimons Fellowship (SPB), a Johns Hopkins Science of Learning Grant (SPB, LAG), National Science Foundation Grant 1656592 (SPB, LAG), NIH training grant T32 GM07814 (JDR), a BoerhingerIngelheim Fonds Fellowship (MC), and the NINDS (NS050274). 
Figure 1: Layer 6 corticothalamic neuron subclasses distinguished by their axonal projections

809 have distinct gene expression profiles. $(\mathbf{a}, \mathbf{f})$ The labeling schemes for two classes of layer 6

810 corticothalamic neurons (L6CThNs; VPM: Ventral posterior medial nucleus; POm: Posterior

811 medial nucleus). (b) Injection of a retrograde tracer (red, Alexa 555-Cholera toxin B) into VPM in

812 an Ntsr1-Cre;YFP mouse. Low (c) and high (d) magnification images of layer 6 (L6) of barrel

813 cortex (BC) showing colocalization of the retrograde tracer (red) and YFP (green). (e)

814 Quantification of the colocalization (mean $\pm S E ; n=4$ mice). (g) Injection of a retrograde tracer

815 (red) in POm of an Ntsr1-Cre;YFP mouse. Low (h) and high (i) magnification images of BC

816 showing the colocalization of the tracer to CThNs in lower L6. (j) Quantification of the

817 colocalization (mean $\pm S E ; n=3$ mice). (k) Matrix showing the 69 genes differentially expressed

818 between pools of VPM/POm and VPM-only L6CThNs from three replicates. Scale bars, $(\mathbf{b}, \mathbf{g}) 500$

$819 \mu \mathrm{m} ;(\mathbf{c}, \mathbf{h}) 50 \mu \mathrm{m} ;$ and $(\mathbf{d}, \mathbf{i}) 10 \mu \mathrm{m}$.

821 Figure 2: Unbiased clustering of single transcriptomes of layer 6 corticothalamic neurons

822 defines two subtypes with strong axonal projection bias. (a) tSNE plot showing two subtypes of

823 layer 6 corticothalamic neurons (L6CThNs) identified through unbiased clustering of single

824 L6CThN transcriptomes. (b) Same tSNE plot as in (a), with each L6CThN color-coded by its axonal

825 projection label. (c) Fraction of VPM-only (green) and VPM/POm (red) L6CThNs in each

826 transcriptionally defined subtype for each replicate. (d) Matrix showing the hierarchical

827 clustering of the 346 individual L6CThNs ( $\mathrm{x}$-axis) and the 286 genes differentially expressed (DE)

828 between the two subtypes (y-axis, $0.1 \%$ FDR). (e) tSNE plots showing the normalized expression

829 levels of two differentially expressed genes enriched in subtype 1 ( $F x y d 6$, left) or subtype 2 
830 (Lamp5, right). (f) tSNE plots showing the normalized expression levels of two differentially

831 expressed long-noncoding RNAs enriched in subtype 1 (linc-Tmem20, left) or subtype 2 (Pantr1,

832 right). (g) Low magnification image of linc-Tmem20 (red) in barrel cortex of an Ntsr1-

833 Cre;tdTomato (green) mouse using a combination of in situ hybridization (linc-Tmem20) and

834 immunohistochemistry (tdTomato). Insets show higher expression of linc-Tmem20 in L6CThNs in

835 lower layer 6 (L6, Inset 2) relative to upper L6 (Inset 1). (h) LacZ expression in barrel cortex of a

836 heterozygous Pantr1-LacZ mouse following injections of green retrograde tracer in VPM and red

837 tracer in POm. Insets show puncta of LacZ in VPM-only L6CThNs (Column 1, green) and not in

838 VPM/POm L6CThNs (Column 2, red and green L6CThNs). (i) Barplot showing the fraction of

839 VPM-only and VPM/POm L6CThNs expressing LacZ (mean \pm SE; $n=3$ mice). (j) Boxplot of the

840 median number of genes detected across all cells for each subtype by replicate pair. (Replicate

841 1: Median number of genes detected: Subtype 1: $5582 \pm 526.3$ (sd), Subtype 2: $5080 \pm 650.0$

842 (sd), $p<2.169 \times 10^{-10}$, Mann-Whitney test; Replicate 2: Median number of genes detected:

843 Subtype 1: $6950 \pm 545.4(\mathrm{sd})$, Subtype 2: $6569 \pm 478.7(\mathrm{sd}), \mathrm{p}<7.071 \times 10^{-7}$, Mann-Whitney test).

844 (k) Cumulative probability distribution of the pairwise Euclidean distances among cells in

845 subtype 1 (gold) and subtype 2 (blue) $\left(p<2.2 \times 10^{-16}\right.$, Welch's two-sample t-test). The black line

846 represents the pairwise distances among a random sample of 100 cells drawn from the 346

847 cells. 95\% confidence interval is shown in light grey (Dvoretzky-Kiefer-Wolfowitz inequality).

848 Scale bars, (g) $100 \mu \mathrm{m}, 20 \mu \mathrm{m}$; (h) $20 \mu \mathrm{m}, 5 \mu \mathrm{m}$.

850 Figure 3: Coordinately regulated gene sets contribute to the transcriptional identities of layer

8516 corticothalamic neuron subtypes. (a) Weighted gene co-expression network analysis

852 (WGCNA) on variance-stabilized gene expression estimates identifies modules of coordinately 
853 regulated genes, which are grouped using hierarchical clustering of module eigengenes. (b)

854 Pearson correlation of each module eigengene with parameters for both transcriptional subtype

855 and label. Significance of each correlation was determined $\left({ }^{*}\right)$ using the Pearson's product

856 moment test ( $p<0.01$; Benjamini-Hochberg corrected). (c) Pearson correlation of each module

857 eigengene with component rotations for principal components 1-5. (d,e) Hypergeometric test

858 for enrichment of the 286 genes differentially expressed between transcriptionally defined

859 L6CThN subtypes (d) and genes associated with neuronal activity (e) within each module ( $p<$

$860 \quad 0.01$; Benjamini-Hochberg corrected).

862 Figure 4: Variation in the transcriptional profiles of layer 6 corticothalamic neurons is defined

863 by both subtype-specific genes and genes reflecting laminar location within layer 6 . (a,d) tSNE

864 plots showing the eigenvalue for each cell for the two WGCNA modules most correlated with

865 the first principal component (a, Midnight Blue; d, Turquoise). (b,c,e,f) Gene expression tSNE

866 plots (left) showing the normalized gene expression level in each cell for representative genes

867 with significant weights on PC1. (b) and (c) are representative members of the Midnight Blue

868 module, and (e) and (f) belong to the Turquoise module. Single molecule fluorescence in situ

869 hybridization (smFISH, middle) showing images of mRNAs detected for each gene of interest

870 (magenta), tdTomato (green), NeuN (cyan), and DAPI (blue) in layer 6 of Ntsr1-Cre;tdTomato

871 mice. Quantitative gene expression analysis of smFISH (right) showing the number of mRNAs

872 expressed per neuron as a function of normalized vertical position in layer 6 and neuronal cell

873 type (L6CThNs: Ntsr1;tdTomato-positive;NeuN-positive neurons in green and non-L6CThNs:

874 Ntsr1;tdTomato-negative, NeuN-positive neurons in grey). Curves represent Loess fits to the

875 individual data points, grouped by cell type, and shaded areas correspond to $95 \%$ confidence 
876 intervals. Statistics: (b, Lamp5) "Subtype specific" $p<7.3231 \times 10^{-19} ;$ "CThN+ position specific" p

$877<1.175 \times 10^{-17} ;$ "CThN- position specific" p < 0.0035; (c, Pantr1) "Subtype specific" $p<0.9931$;

878 "CThN+ position specific" $p<9.243 \times 10^{-11} ;$ "CThN- position specific" $p<1.021 \times 10^{-14}$; (e,

879 Serpini1) "Subtype specific" $p<1.606 \times 10^{-06} ;$ "CThN+ position specific" $p<1.045 \times 10^{-06}$; "CThN-

880 position specific" $\mathrm{p}<0.3342 ;$ (f, Gabra5) "Subtype specific" $\mathrm{p}<0.1020$; "CThN+ position

881 specific" $p<1.994 \times 10^{-3} ;$ “CThN-position specific” $p<0.09873$.

883 Figure 5: A signature of neuronal activity significantly contributes to the transcriptional

884 identities of layer 6 corticothalamic neuron subtypes. (a) Module eigengenes for the four

885 modules with significant enrichment for genes associated with neuronal activity. (b,c) tSNE plots

886 showing the discretized expression of genes with the highest (b) and second highest (c) weights

887 in each direction of PC1. (d,e) tSNE plots showing the discretized expression of genes with the

888 highest (d) and second highest (e) weights in each direction of PC2. (f,g) tSNE plots showing the

889 discretized expression levels of four genes reflecting neuronal activity: Bdnf and Camk2n1 (f),

890 and Fos and Plk5 (g).

891

892 Figure 6: Sustained modulation of gene expression after sensory manipulation in layer 6

893 corticothalamic neurons. (a) Experimental design for sensory manipulation and single-cell

894 transcriptional analysis of L6CThNs from the barrel cortex at 1 and 7 days post manipulation. (b)

895 tSNE plot of all 1023 neurons obtained from baseline (Day 0), 1, and 7 days following sensory

896 manipulation using the 286 genes identified as differentially expressed between L6CThN

897 subtypes at day 0. Day 0 neurons are labeled by transcriptional subtype. Day 1 and Day 7

898 neurons are colored light gray. tSNE positions were fit to a Gaussian mixture model (black lines) 
899 which was used to classify Day 1 and Day 7 neurons as members of the previously defined

900 L6CThN transcriptional subtypes. (c) tSNE plot colored by transcriptional subtype as assigned in

901 (b). 10 of 340 neurons (2.9\%) from Day 0 were assigned to a different subtype than in Fig. 2a

902 and are labeled light green. (d) tSNE plot with neurons colored by projection label. (e) k-means

903 clustering analysis of mean-centered gene expression, aggregated by Day and transcriptional

904 subtype (Subtype 1: gold; Subtype 2: blue) for genes with significant differential expression after

905 sensory manipulation. Semi-transparent lines represent individual genes and bold lines

906 represent cluster centroids.

Figure 7: Pseudotemporal reconstruction of transcriptional responses to sensory manipulation

909 in layer 6 corticothalamic neurons. (a) Discriminative dimensionality reduction projection of

9101023 L6CThNs using genes identified as significantly differentially expressed after sensory

911 manipulation. Neurons are colored by day relative to manipulation. (b) Density distribution of

912 L6CThNs across pseudotime, grouped by day following manipulation. (c) Heatmap of normalized

913 response curves for the 1507 genes with significant differential expression across pseudotime,

914 and significantly enriched gene sets identified for each cluster $\left(p<1.0 \times 10^{-2}\right.$, hypergeometric

915 test).

916

917 Figure 8: Sensory manipulation induces distinct cellular responses in layer 6 corticothalamic

918 neurons biased with respect to transcriptional subtype. (a) Distribution of the pairwise

919 Euclidean distances within each subtype for subtype 1 (left, gold) and subtype 2 (right, blue),

920 using variance-stabilized expression estimates for all expressed genes, showing a significant

921 increase in heterogeneity of gene expression for both subtype 1 and subtype 2. (b) Distribution 
922 of pairwise inter-subtype Euclidean distances between transcriptionally defined L6CThN

923 subtypes across all expressed genes plotted for each day following sensory manipulation. There

924 is a significant divergence between these two subtypes across time points as indicated by a

925 positive shift in the distances after induction of experience-dependent plasticity. (c) Density

926 distributions of L6CThNs at each day plotted across pseudotime for the two transcriptional

927 subtypes of L6CThNs. (d) Discriminative dimensionality reduction projection of 1023 L6CThNs

928 shown in Fig. 7a, now colored by transcriptional subtype. Red and blue arrows indicate the first

929 major cellular response branch point following sensory manipulation. Grey arrow indicates the

930 tree root state and direction of response progression. Pie charts indicate the proportion of each

931 subtype in the population for each branch. (e) BEAM analyses of gene sets with significant

932 differential expression dependent on either major branch point showing all significant DE genes.

933 (f-h) BEAM heatmap for branch-dependent transcription factors not detected in the aggregate

934 pseudotime response (f), presynaptic proteins ( $\mathbf{g})$, and ligand-gated neurotransmitter receptors

935 (h).

937 SUPPLEMENTARY INFORMATION (provided separately) 


\section{REFERENCES}

940 1. Junker, J.P. \& van Oudenaarden, A. Every cell is special: genome-wide studies add a new

941 dimension to single-cell biology. Cell 157, 8-11 (2014).

942 2. Liu, S. \& Trapnell, C. Single-cell transcriptome sequencing: recent advances and remaining

$943 \quad$ challenges. F1000Res 5, 182 (2016).

944 3. Molyneaux, B.J., Arlotta, P., Menezes, J.R. \& Macklis, J.D. Neuronal subtype specification in

945 the cerebral cortex. Nat Rev Neurosci 8, 427-437 (2007).

946 4. West, A.E. \& Greenberg, M.E. Neuronal activity-regulated gene transcription in synapse

947 development and cognitive function. Cold Spring Harb Perspect Biol 3 (2011).

948 5. Lyons, M.R. \& West, A.E. Mechanisms of specificity in neuronal activity-regulated gene

$949 \quad$ transcription. Prog Neurobiol 94, 259-295 (2011).

950 6. Flavell, S.W. \& Greenberg, M.E. Signaling mechanisms linking neuronal activity to gene

951 expression and plasticity of the nervous system. Annu Rev Neurosci 31, 563-590 (2008).

952 7. Greig, L.C., Woodworth, M.B., Galazo, M.J., Padmanabhan, H. \& Macklis, J.D. Molecular logic

953 of neocortical projection neuron specification, development and diversity. Nat Rev Neurosci

$954 \quad 14,755-769$ (2013).

955 8. Molyneaux, B.J., et al. DeCoN: genome-wide analysis of in vivo transcriptional dynamics

956 during pyramidal neuron fate selection in neocortex. Neuron 85, 275-288 (2015).

957 9. Darmanis, S., et al. A survey of human brain transcriptome diversity at the single cell level.

$958 \quad$ Proc Natl Acad Sci U S A 112, 7285-7290 (2015).

959 10. Lake, B.B., et al. Neuronal subtypes and diversity revealed by single-nucleus RNA sequencing 960 of the human brain. Science 352, 1586-1590 (2016).

961 11. Pollen, A.A., et al. Low-coverage single-cell mRNA sequencing reveals cellular heterogeneity 
and activated signaling pathways in developing cerebral cortex. Nat Biotechnol 32, $1053-$

$9631058(2014)$

964 12. Tasic, B., et al. Adult mouse cortical cell taxonomy revealed by single cell transcriptomics.

$965 \quad$ Nat Neurosci 19, 335-346 (2016).

966 13. Zeisel, A., et al. Brain structure. Cell types in the mouse cortex and hippocampus revealed by

967 single-cell RNA-seq. Science 347, 1138-1142 (2015).

968 14. Hevner, R.F., et al. Beyond laminar fate: toward a molecular classification of cortical

969 projection/pyramidal neurons. Dev Neurosci 25, 139-151 (2003).

970 15. Sugino, K., et al. Molecular taxonomy of major neuronal classes in the adult mouse

971 forebrain. Nat Neurosci 9, 99-107 (2006).

972 16. Sorensen, S.A., et al. Correlated gene expression and target specificity demonstrate

973 excitatory projection neuron diversity. Cereb Cortex 25, 433-449 (2015).

974 17. Sanes, J.R. \& Masland, R.H. The types of retinal ganglion cells: current status and

975 implications for neuronal classification. Annu Rev Neurosci 38, 221-246 (2015).

976 18. Wagner, A., Regev, A. \& Yosef, N. Revealing the vectors of cellular identity with single-cell

977 genomics. Nat Biotechnol 34, 1145-1160 (2016).

978 19. Dueck, H., Eberwine, J. \& Kim, J. Variation is function: Are single cell differences functionally

979 important?: Testing the hypothesis that single cell variation is required for aggregate

$980 \quad$ function. Bioessays 38, 172-180 (2016).

981 20. Bourassa, J. \& Deschenes, M. Corticothalamic projections from the primary visual cortex in

982 rats: a single fiber study using biocytin as an anterograde tracer. Neuroscience 66, 253-263

$983 \quad$ (1995)

984 21. Bourassa, J., Pinault, D. \& Deschenes, M. Corticothalamic projections from the cortical barrel 
985 field to the somatosensory thalamus in rats: a single-fibre study using biocytin as an

986 anterograde tracer. Eur J Neurosci 7, 19-30 (1995).

987 22. Briggs, F., Kiley, C.W., Callaway, E.M. \& Usrey, W.M. Morphological substrates for parallel

988 streams of corticogeniculate feedback originating in both V1 and V2 of the macaque

989 monkey. Neuron 90, 388-399 (2016).

990 23. Briggs, F. \& Usrey, W.M. Parallel processing in the corticogeniculate pathway of the

991 macaque monkey. Neuron 62, 135-146 (2009).

992 24. Katz, L.C. Local circuitry of identified projection neurons in cat visual cortex brain slices. J

$993 \quad$ Neurosci 7, 1223-1249 (1987).

994 25. Killackey, H.P. \& Sherman, S.M. Corticothalamic projections from the rat primary

995 somatosensory cortex. J Neurosci 23, 7381-7384 (2003).

996 26. Kwegyir-Afful, E.E. \& Simons, D.J. Subthreshold receptive field properties distinguish

997 different classes of corticothalamic neurons in the somatosensory system. J Neurosci 29,

$998 \quad 964-972(2009)$.

999 27. Usrey, W.M. \& Fitzpatrick, D. Specificity in the axonal connections of layer VI neurons in tree

1000 shrew striate cortex: Evidence for distinct granular and supragranular systems. J Neurosci

$1001 \quad 16,1203-1218(1996)$.

1002 28. Zhang, Z.W. \& Deschenes, M. Intracortical axonal projections of lamina VI cells of the

1003 primary somatosensory cortex in the rat: a single-cell labeling study. J Neurosci 17, 6365-

$6379(1997)$.

1005 29. Thomson, A.M. Neocortical layer 6, a review. Front Neuroanat 4, 13 (2010).

1006 30. Gong, S., et al. Targeting Cre recombinase to specific neuron populations with bacterial

1007 artificial chromosome constructs. J Neurosci 27, 9817-9823 (2007). 
1008

1009

1010

1011

1012

1013

1014

1015

1016

1017

1018

1019

1020

1021

1022

1023

1024

1025

1026

1027

1028

1029

1030

31. Kim, J., Matney, C.J., Blankenship, A., Hestrin, S. \& Brown, S.P. Layer 6 corticothalamic neurons activate a cortical output layer, layer 5a. J Neurosci 34, 9656-9664 (2014).

32. Bortone, D.S., Olsen, S.R. \& Scanziani, M. Translaminar inhibitory cells recruited by layer 6 corticothalamic neurons suppress visual cortex. Neuron 82, 474-485 (2014).

33. Brennecke, P., et al. Accounting for technical noise in single-cell RNA-seq experiments. Nat Methods 10, 1093-1095 (2013).

34. Hicks, S., Teng, M. \& Irizarry, R.A. On the widespread and critical impact of systematic bias and batch effects in single-cell RNA-seq data. BioRxiv doi:10.1101/025528 (2015).

35. Chung, N.C. \& Storey, J.D. Statistical significance of variables driving systematic variation in high-dimensional data. Bioinformatics 31, 545-554 (2015).

36. Kiselev, V.Y., et al. SC3: consensus clustering of single-cell RNA-seq data. Nat Methods 14, 483-486 (2017).

37. Lin, P., Troup, M. \& Ho, J.W. CIDR: Ultrafast and accurate clustering through imputation for single-cell RNA-seq data. Genome Biol 18, 59 (2017).

38. Lein, E.S., et al. Genome-wide atlas of gene expression in the adult mouse brain. Nature 445, 168-176 (2007).

39. Sauvageau, M., et al. Multiple knockout mouse models reveal lincRNAs are required for life and brain development. Elife 2, e01749 (2013).

40. Joo, K.M., et al. Distribution of vasoactive intestinal peptide and pituitary adenylate cyclaseactivating polypeptide receptors (VPAC1, VPAC2, and PAC1 receptor) in the rat brain. J Comp Neurol 476, 388-413 (2004).

41. Sun, Q.Q., Prince, D.A. \& Huguenard, J.R. Vasoactive intestinal polypeptide and pituitary adenylate cyclase-activating polypeptide activate hyperpolarization-activated cationic 
1031 current and depolarize thalamocortical neurons in vitro. J Neurosci 23, 2751-2758 (2003).

1032 42. Langfelder, P. \& Horvath, S. WGCNA: an R package for weighted correlation network

1033 analysis. BMC Bioinformatics 9, 559 (2008).

1034 43. Lacar, B., et al. Nuclear RNA-seq of single neurons reveals molecular signatures of activation.

$1035 \quad$ Nat Commun 7, 11022 (2016).

1036 44. Mardinly, A.R., et al. Sensory experience regulates cortical inhibition by inducing IGF1 in VIP

1037 neurons. Nature 531, 371-375 (2016).

1038 45. Cho, J.H., Huang, B.S. \& Gray, J.M. RNA sequencing from neural ensembles activated during

1039 fear conditioning in the mouse temporal association cortex. Sci Rep 6, 31753 (2016).

1040 46. Kim, T.K., et al. Widespread transcription at neuronal activity-regulated enhancers. Nature

$1041 \quad 465,182-187(2010)$.

1042 47. Wallace, H. \& Fox, K. The effect of vibrissa deprivation pattern on the form of plasticity

1043 induced in rat barrel cortex. Somatosens Mot Res 16, 122-138 (1999).

1044 48. Fox, K. Barrel Cortex (Cambridge University Press, Cambridge, 2008).

1045 49. Fraley, C., Raftery, A.E., Murphy, T.B. \& Scrucca, L. mclust Version 4 for R: Normal Mixture

1046 Modeling for Model-Based Clustering, Classification, and Density Estimation Technical

1047 Report No. 597. Department of Statistics, University of Washington (2012).

1048 50. Fraley, C. \& Raftery, A.E. Model-based clustering, discriminant analysis and density

1049 estimation. Journal of the American Statistical Association 97, 611-631 (2002).

1050 51. Ehlers, M.D. Activity level controls postsynaptic composition and signaling via the ubiquitin-

1051 proteasome system. Nat Neurosci 6, 231-242 (2003).

1052 52. Spiegel, I., et al. Npas4 regulates excitatory-inhibitory balance within neural circuits through

1053 cell-type-specific gene programs. Cell 157, 1216-1229 (2014). 
1054 53. Whitney, O., et al. Core and region-enriched networks of behaviorally regulated genes and

1055 the singing genome. Science 346, 1256780 (2014).

1056 54. Frenkel, M.Y. \& Bear, M.F. How monocular deprivation shifts ocular dominance in visual

1057 cortex of young mice. Neuron 44, 917-923 (2004).

1058 55. Glazewski, S., Benedetti, B.L. \& Barth, A.L. Ipsilateral whiskers suppress experience-

1059 dependent plasticity in the barrel cortex. J Neurosci 27, 3910-3920 (2007).

1060 56. Madisen, L., et al. A robust and high-throughput Cre reporting and characterization system

1061 for the whole mouse brain. Nat Neurosci 13, 133-140 (2010).

1062 57. Schindelin, J., et al. Fiji: an open-source platform for biological-image analysis. Nat Methods

10639 9, 676-682 (2012).

1064 58. Agmon, A. \& Connors, B.W. Thalamocortical responses of mouse somatosensory (barrel)

1065 cortex in vitro. Neuroscience 41, 365-379 (1991).

1066 59. Saxena, A., et al. Trehalose-enhanced isolation of neuronal sub-types from adult mouse

1067 brain. Biotechniques 52, 381-385 (2012).

1068 60. Picelli, S., et al. Full-length RNA-seq from single cells using Smart-seq2. Nat Protoc 9, 171-

$1069181(2014)$.

1070 61. Mudge, J.M. \& Harrow, J. Creating reference gene annotation for the mouse C57BL6/J

1071 genome assembly. Mamm Genome 26, 366-378 (2015).

1072 62. Blackshaw, S. High-throughput RNA in situ hybridization in mouse retina. Methods Mol Biol

$1073935,215-226(2013)$.

1074 63. Goff, L.A., et al. Spatiotemporal expression and transcriptional perturbations by long

1075 noncoding RNAs in the mouse brain. Proc Natl Acad Sci U S A 112, 6855-6862 (2015).

1076 64. Mueller, F., et al. FISH-quant: automatic counting of transcripts in 3D FISH images. Nat 
Methods 10, 277-278 (2013).

1078 65. Li, X., Glazewski, S., Lin, X., Elde, R. \& Fox, K. Effect of vibrissae deprivation on follicle

1079 innervation, neuropeptide synthesis in the trigeminal ganglion, and S1 barrel cortex

1080 plasticity. J Comp Neurol 357, 465-481 (1995).

1081 66. Kim, D., Langmead, B. \& Salzberg, S.L. HISAT: a fast spliced aligner with low memory requirements. Nat Methods 12, 357-360 (2015).

67. Trapnell, C., et al. Differential analysis of gene regulation at transcript resolution with RNAseq. Nat Biotechnol 31, 46-53 (2013).

68. Trapnell, C., et al. Differential gene and transcript expression analysis of RNA-seq experiments with TopHat and Cufflinks. Nat Protoc 7, 562-578 (2012).

69. Trapnell, C., et al. The dynamics and regulators of cell fate decisions are revealed by pseudotemporal ordering of single cells. Nat Biotechnol 32, 381-386 (2014).

70. Huber, W., et al. Orchestrating high-throughput genomic analysis with Bioconductor. Nat Methods 12, 115-121 (2015).

71. Wood, S.N. Fast stable restricted maximum likelihood and marginal likelihood estimation of semiparametric generalized linear models. Journal of the Royal Statistical Society: Series B (Statistical Methodology) 73, 3-36 (2011).

1094 72. Krijthe, J. Rtsne: T-distributed stochastic neighbor embedding using Barnes-Hus

1095 implementation. $\mathrm{R}$ package version 0.10. https://CRAN.R-project.org/package=Rtsne. 1096 (2015).

1097 73. Macosko, E.Z., et al. Highly parallel genome-wide expression profiling of individual cells $1098 \quad$ using nanoliter droplets. Cell 161, 1202-1214 (2015).

1099 74. Ashburner, M., et al. Gene ontology: tool for the unification of biology. The Gene Ontology 
$1100 \quad$ Consortium. Nat Genet 25, 25-29 (2000).

1101 75. Yu, G. \& He, Q.Y. ReactomePA: an R/Bioconductor package for reactome pathway analysis

1102 and visualization. Mol Biosyst 12, 477-479 (2016).

1103 76. Yu, G., Wang, L.G., Han, Y. \& He, Q.Y. clusterProfiler: an R package for comparing biological

1104 themes among gene clusters. OMICS 16, 284-287 (2012).

1105 77. Kolde, R. pheatmap: Pretty heatmaps. R package verion 1.0.8. https://CRAN.R-

1106 project.org/package=pheatmap. (2015).

1107 78. Subramanian, A., et al. Gene set enrichment analysis: a knowledge-based approach for

1108 interpreting genome-wide expression profiles. Proc Natl Acad Sci U S A 102, 15545-15550

$1109 \quad$ (2005). 
a bioRxiv preprint $\mathrm{d} \boldsymbol{b}$
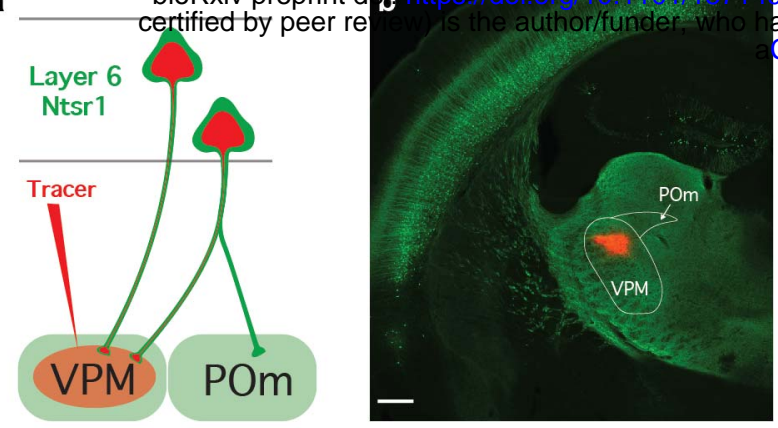

f
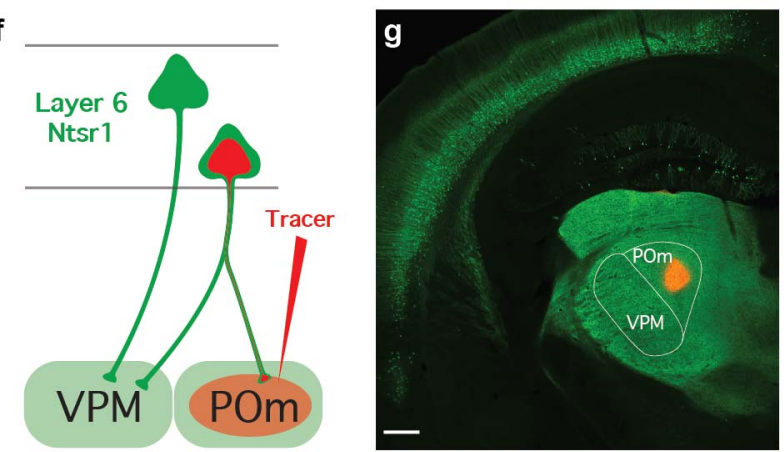
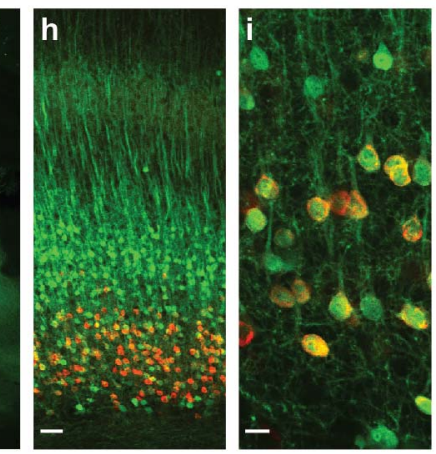

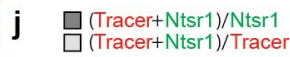

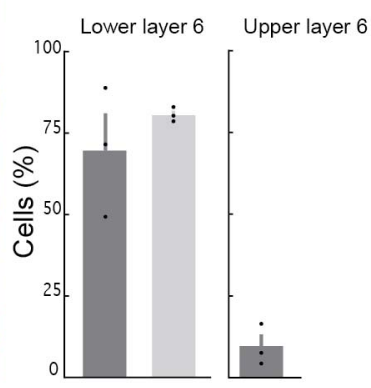

Row Z-score

2

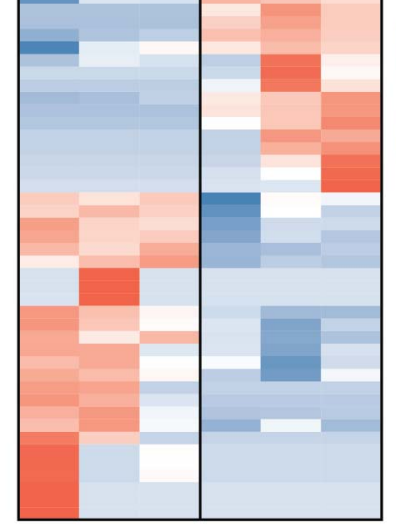

Figure 1

Chevée et al. 
bioRxiv preprint doi: https://doi org/10.1101/157149; this version posted June 30, 2017. The copyright holder for this preprint (which was not certified by peer review) is the author/funder, who has granted bioRxiv a license to display the preprint in perpetuity. It is made available under aCC-BY-NC-ND 4.0 International license.

a

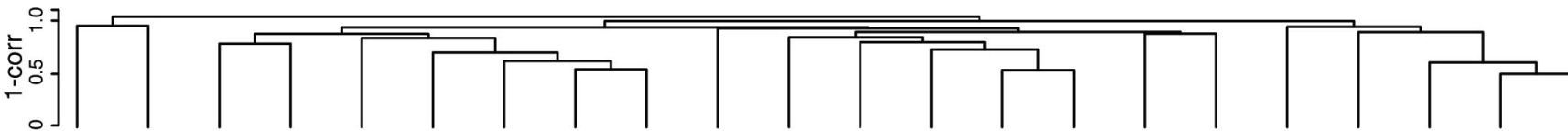

b

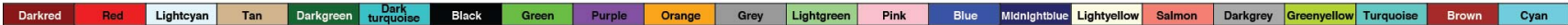

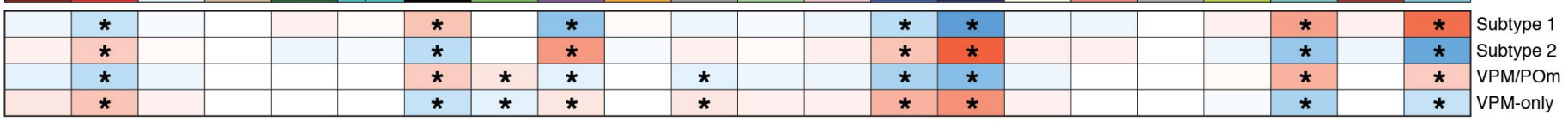

C

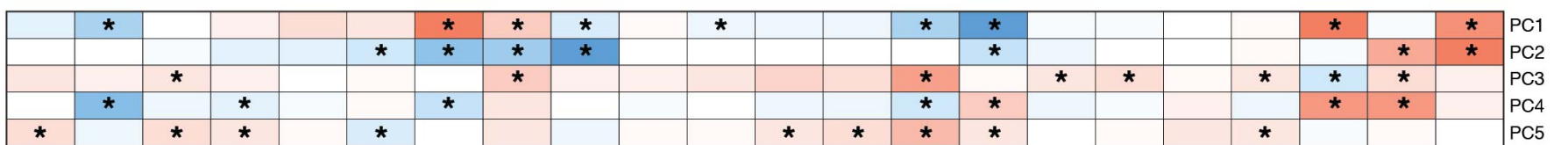

d

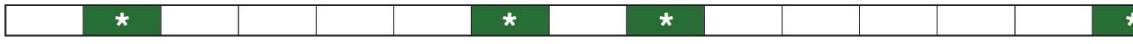

e 
bioRxiv preprint doi: https://doi.org/10.1101/157149; this version posted June 30, 2017. The copyright holder for this preprint (which was not certified by peer review) is the author/funder, who has granted bioRxiv a license to display the preprint in perpetuity. It is made available under

a

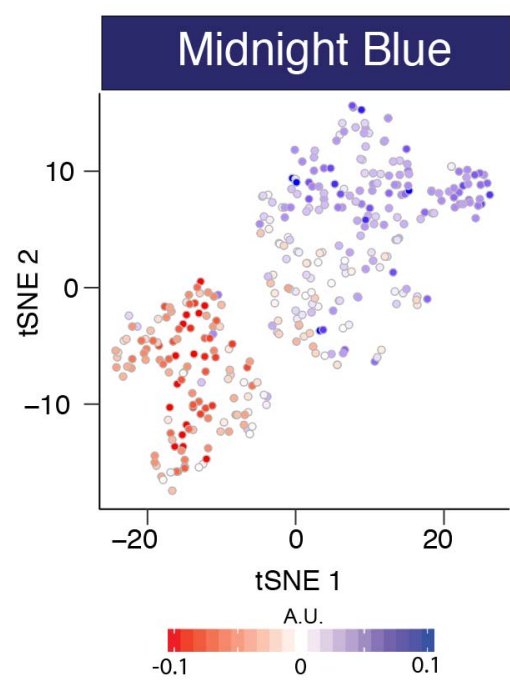

d

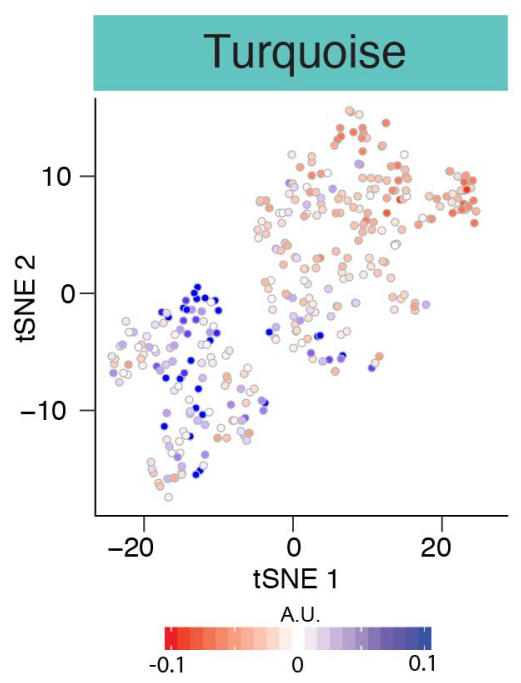

b

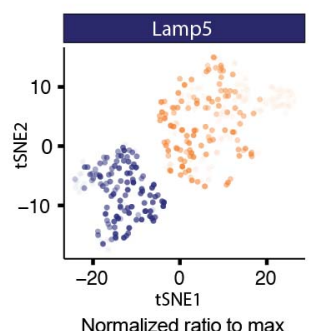

C Normalized ratio to 0
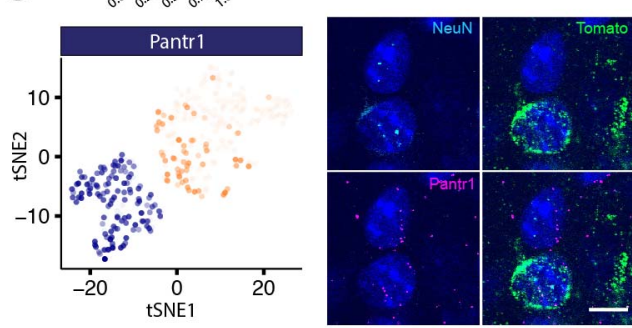

e

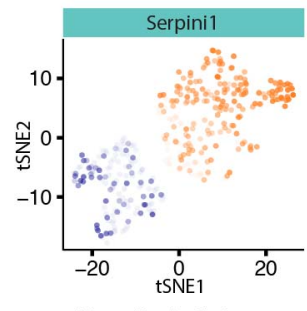

Normalized ratio to max
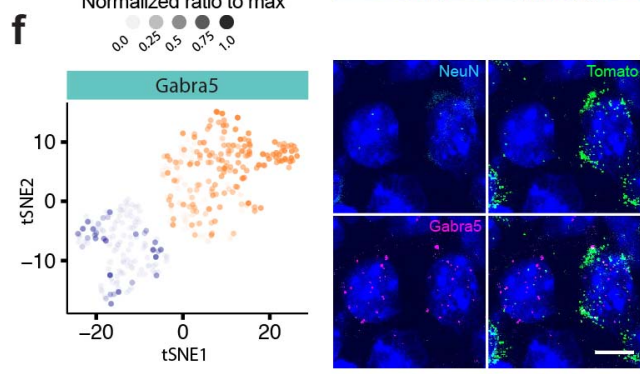
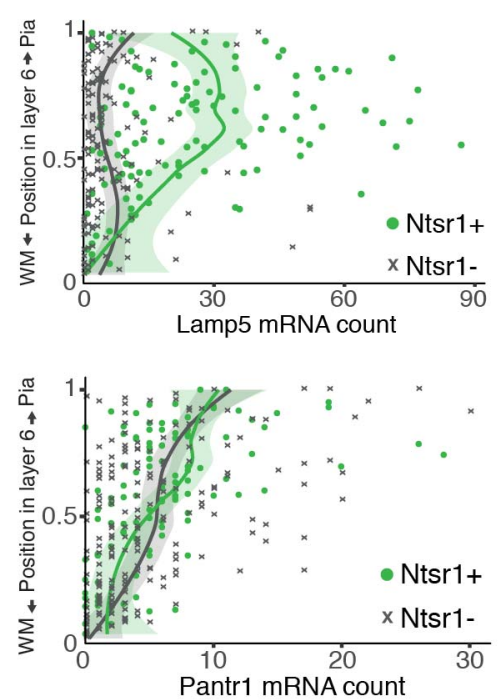

Pantr1 mRNA count
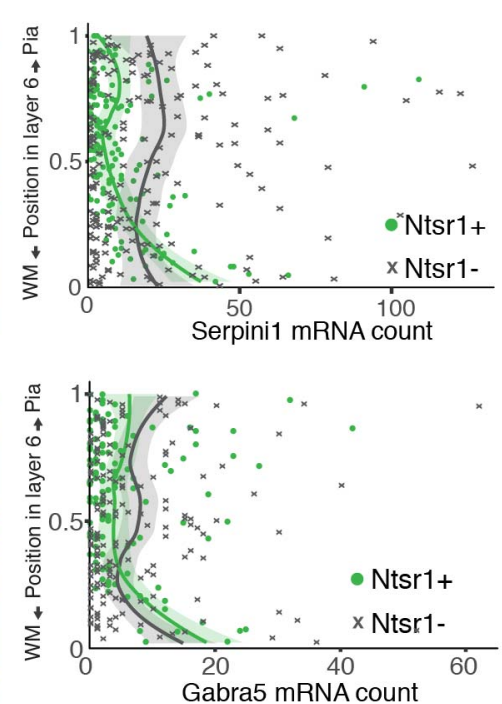

Figure 4

Chevée et al. 
bioRxiv preprint doi: https://doi.org/10.1101/157149; this version posted June 30, 2017. The copyright holder for this preprint (which was not certified by peer review) is the author/funder, who has granted bioRxiv a license to display the preprint in perpetuity. It is made available under aCC-BY-NC-ND 4.0 International license.

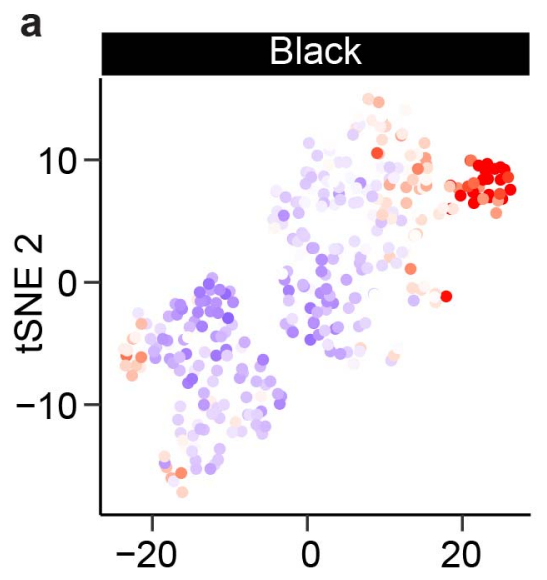

b

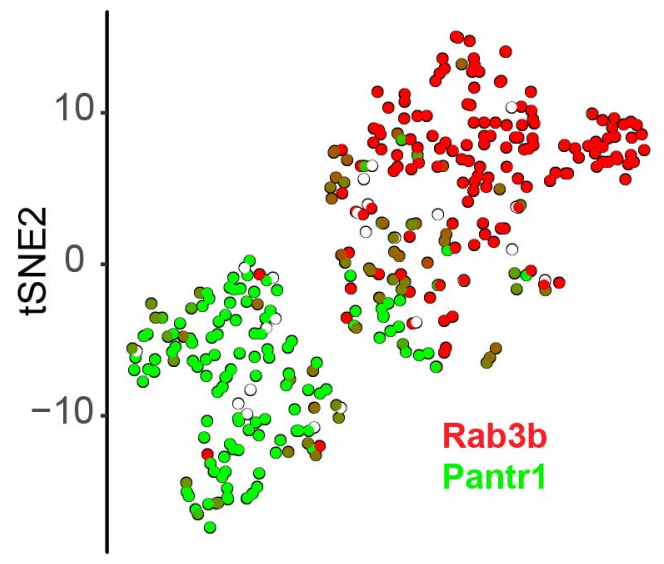

C

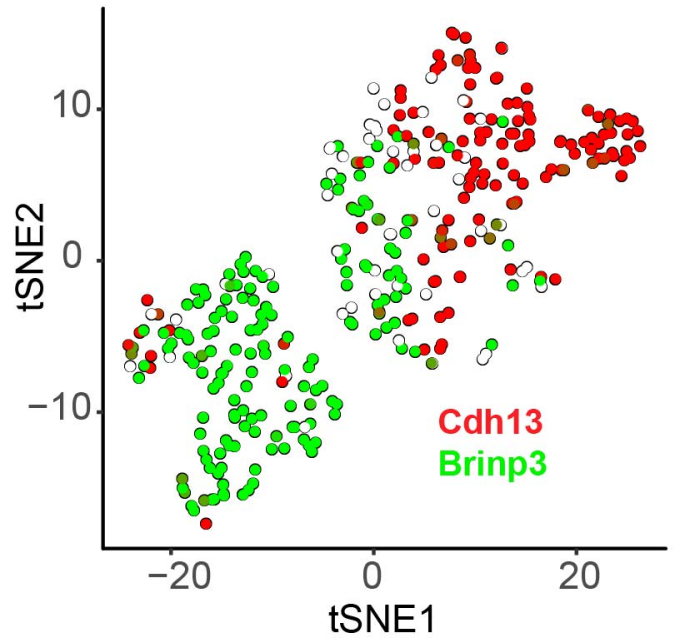

\section{Green}
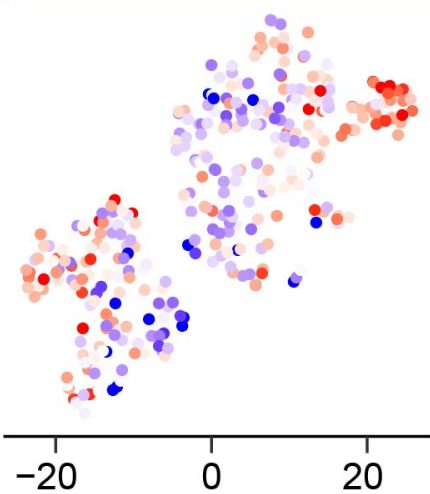

d

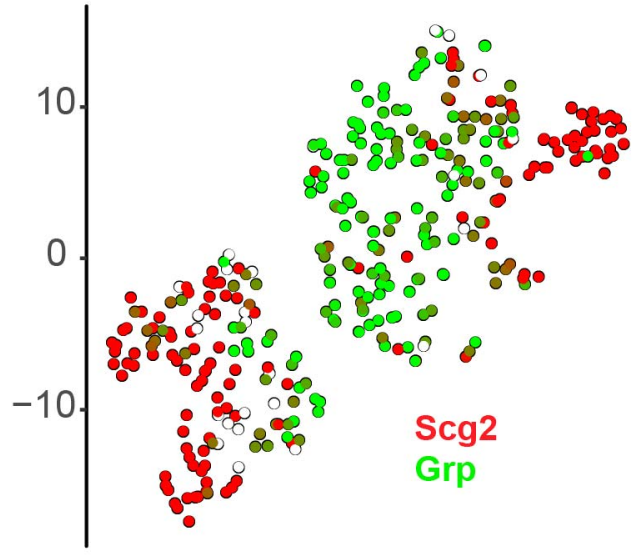

e

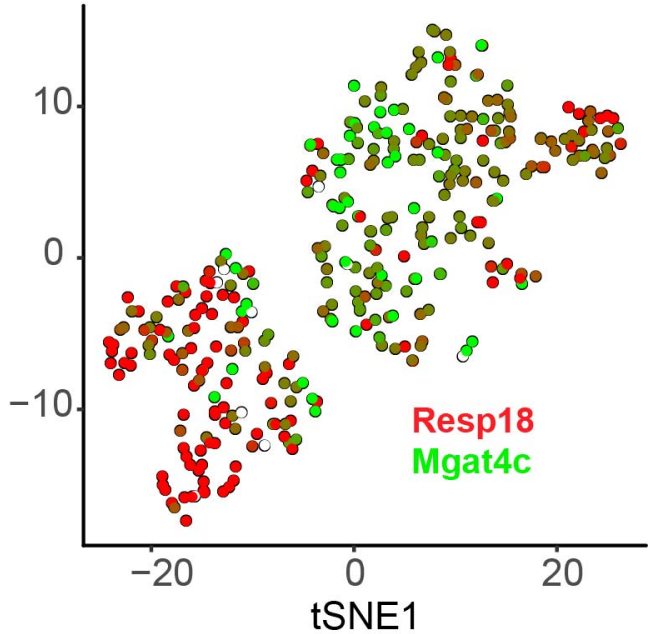

Midnight Blue

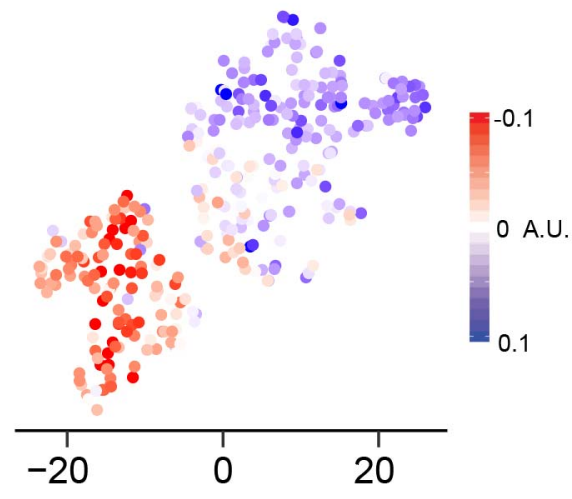

f

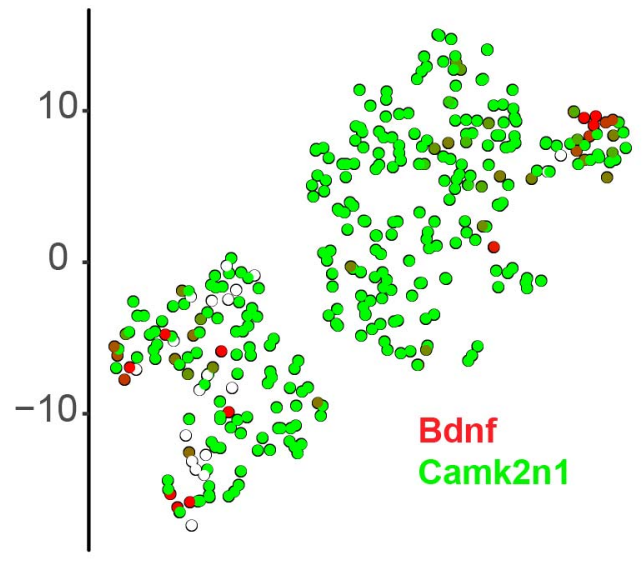

g

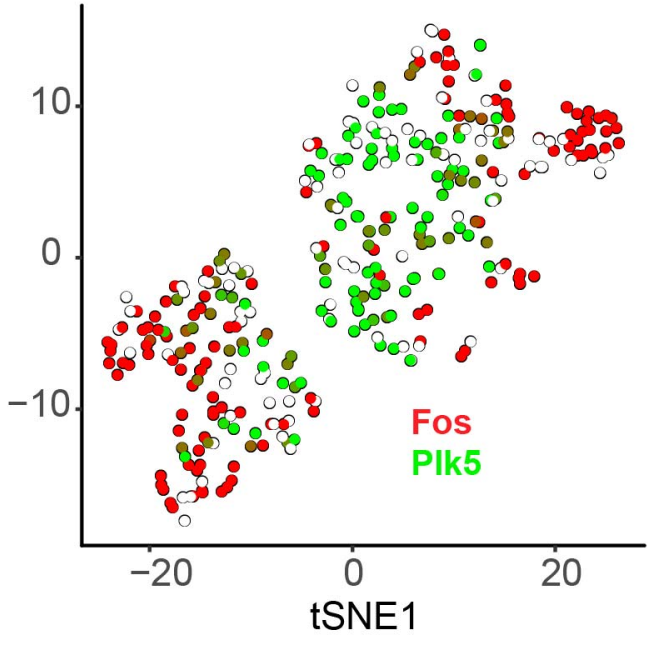

Figure 5

Chevée et al. 
bioRxiv preprint doi: https://doi. org/10.1101/157149; this version posted June 30, 2017. The copyright holder for this preprint (which was not certified by peer review) is the author/funder, who has granted bioRxiv a license to display the preprint in perpetuity. It is made available under

a
1. Label neurons
2. Induce plasticity
3. Collect tissue

Retrobeads $\mathrm{POm} \mathrm{POm}^{\mathrm{O}}$

Ntsr1-Cre:LSL-tdTomato
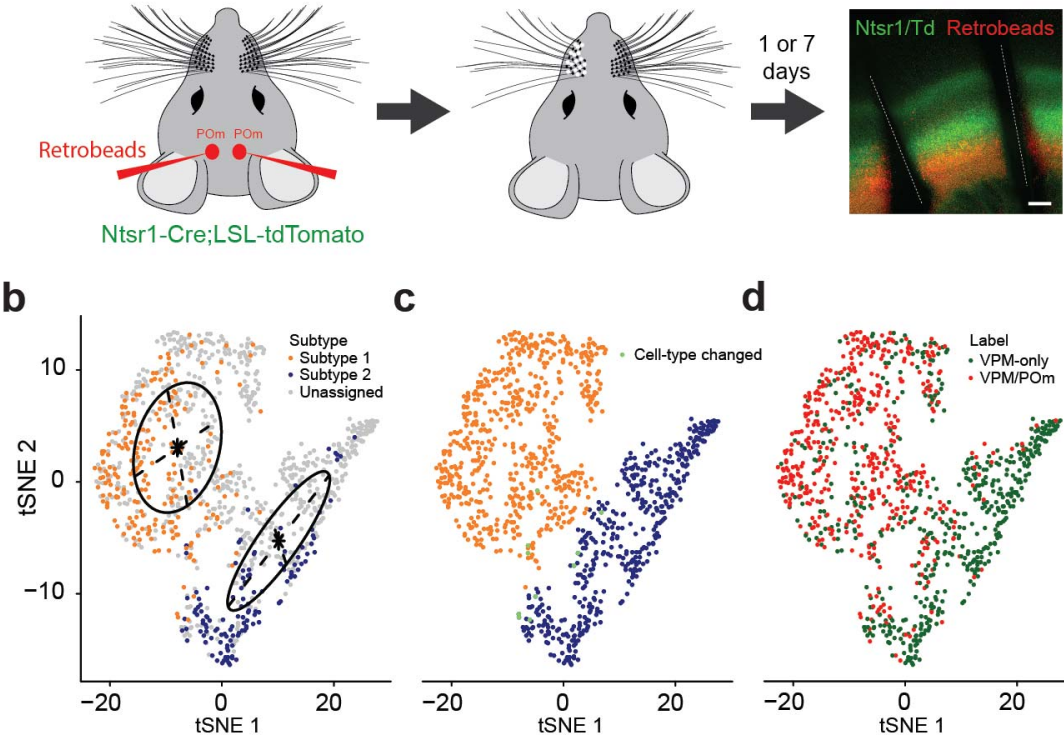

C

d
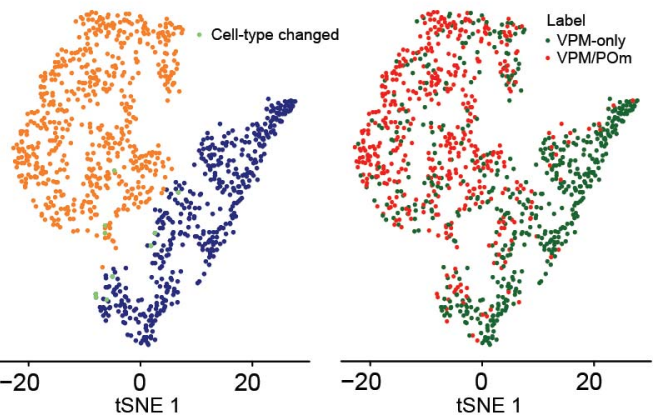

e
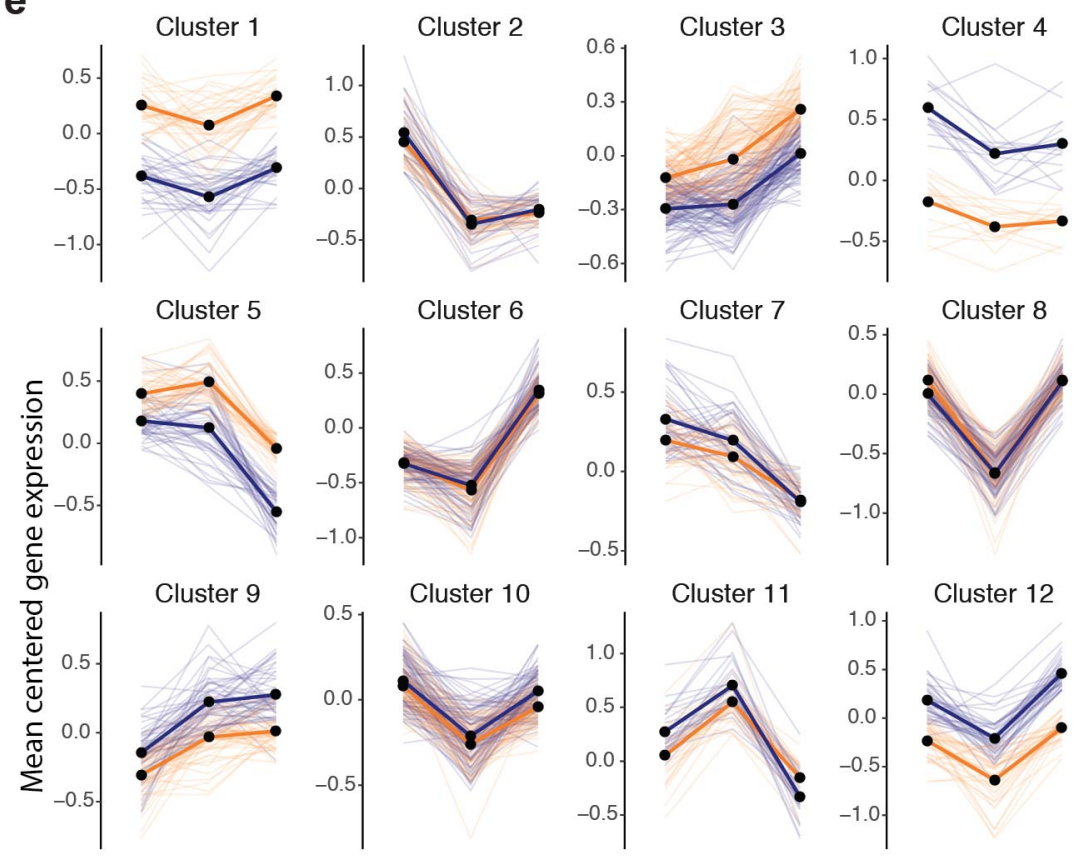

Cluster 6

Cluster 7

Cluster 8
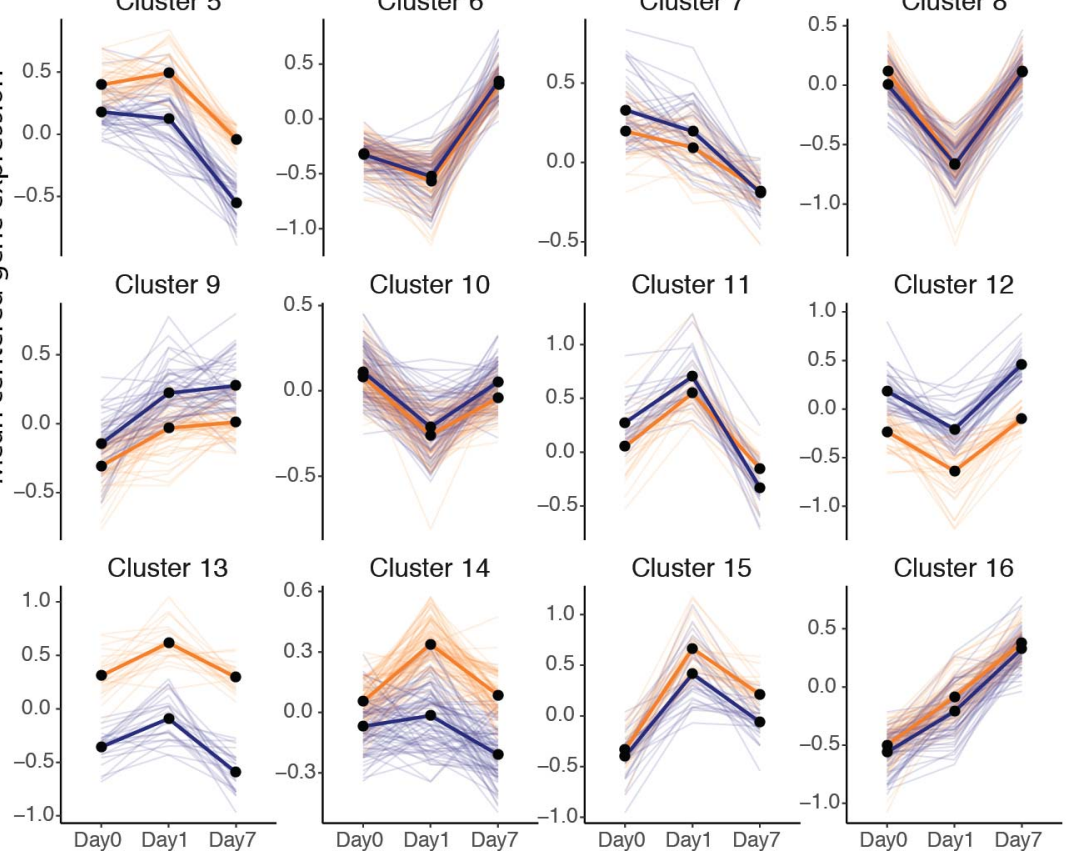
bioRxiv preprint doi: https://doi.org/10.1101/157149; this version posted June 30, 2017. The copyright holder for this preprint (which was not certified by peer review) is the author/funder, who has granted bioRxiv a license to display the preprint in perpetuity. It is made available under aCC-BY-NC-ND 4.0 International license.

a

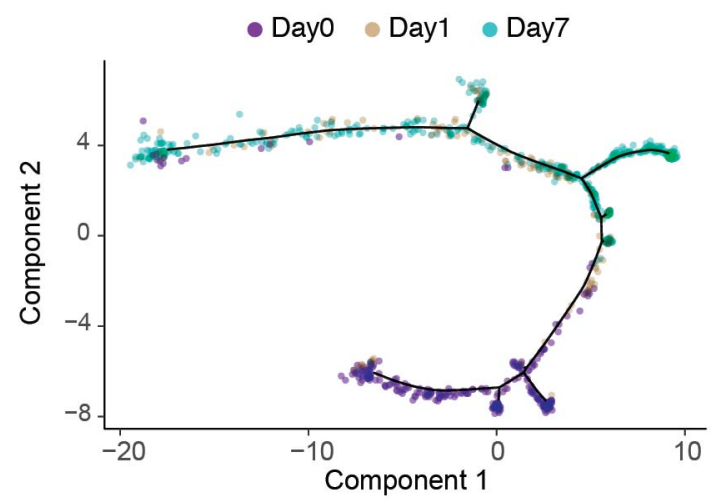

b

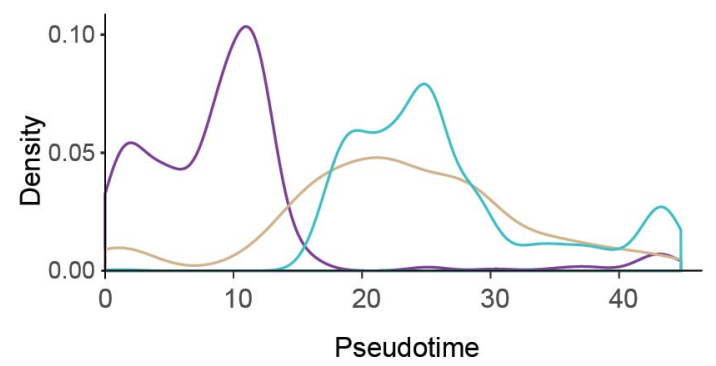

C

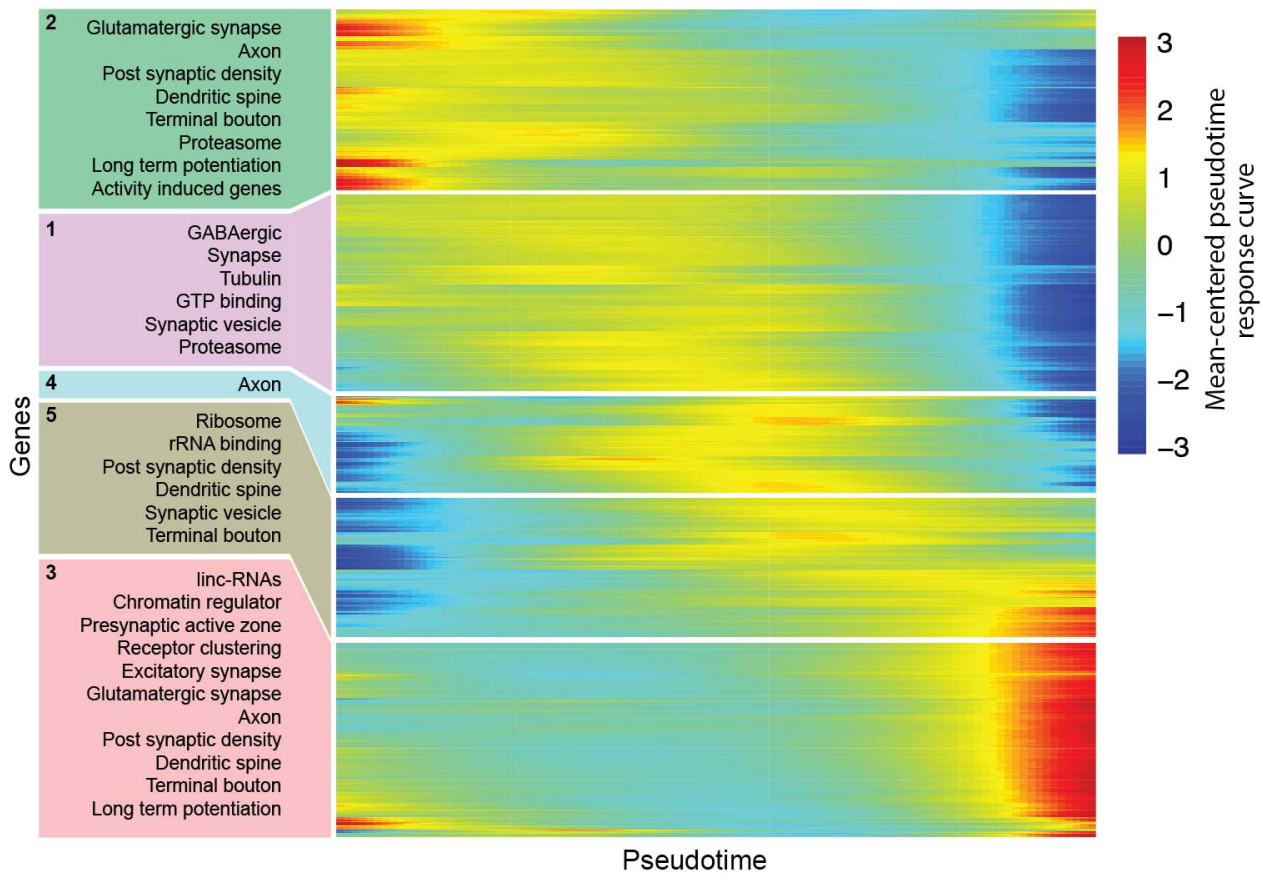

Figure 7

Chevée et al. 


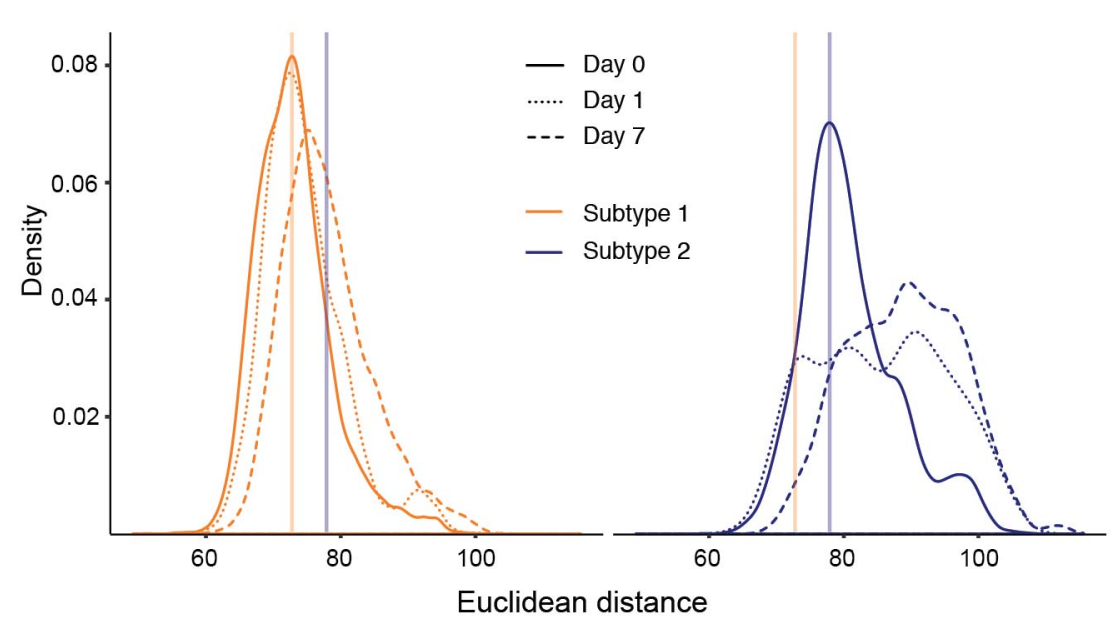

C
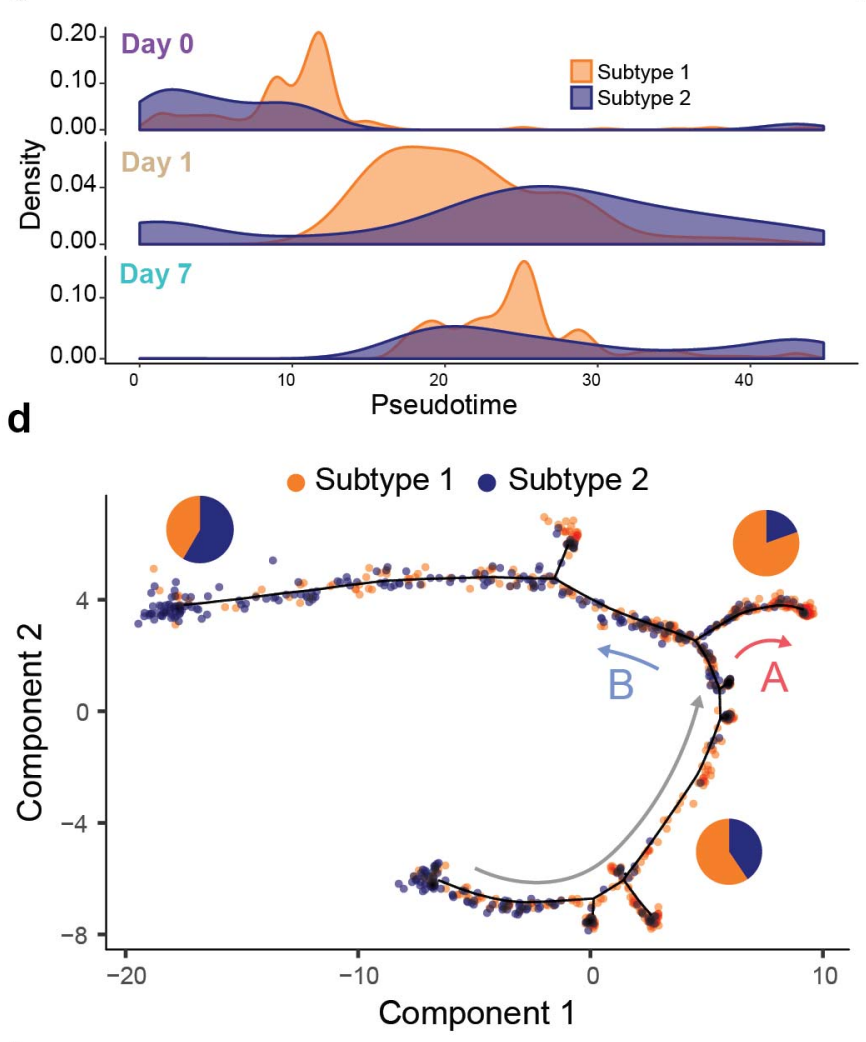

f

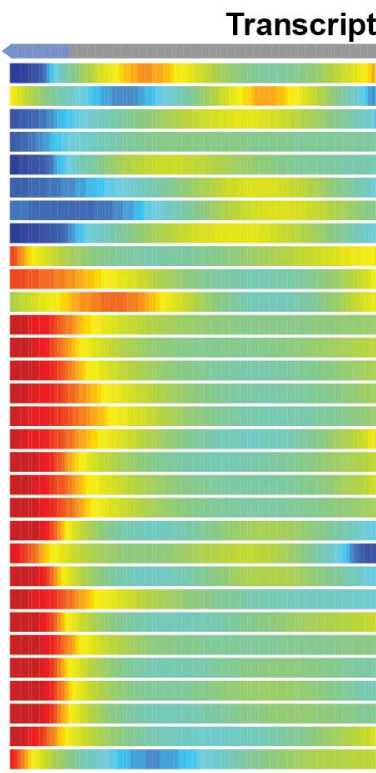

e

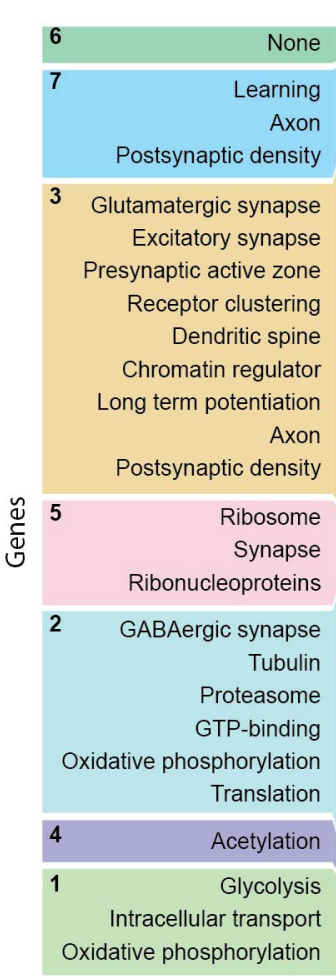

g

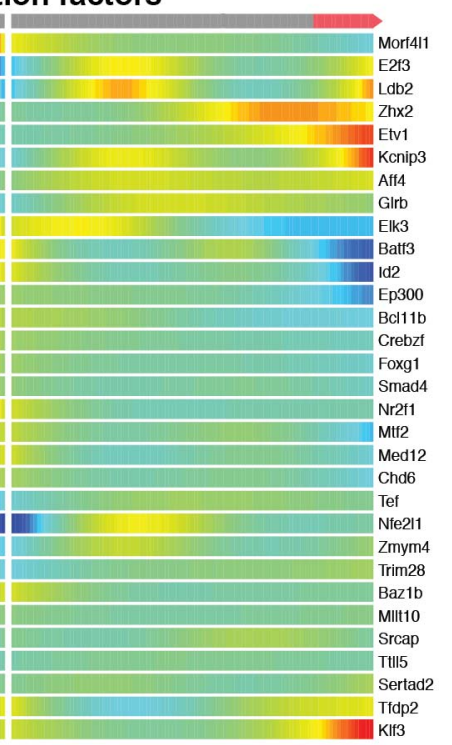

h
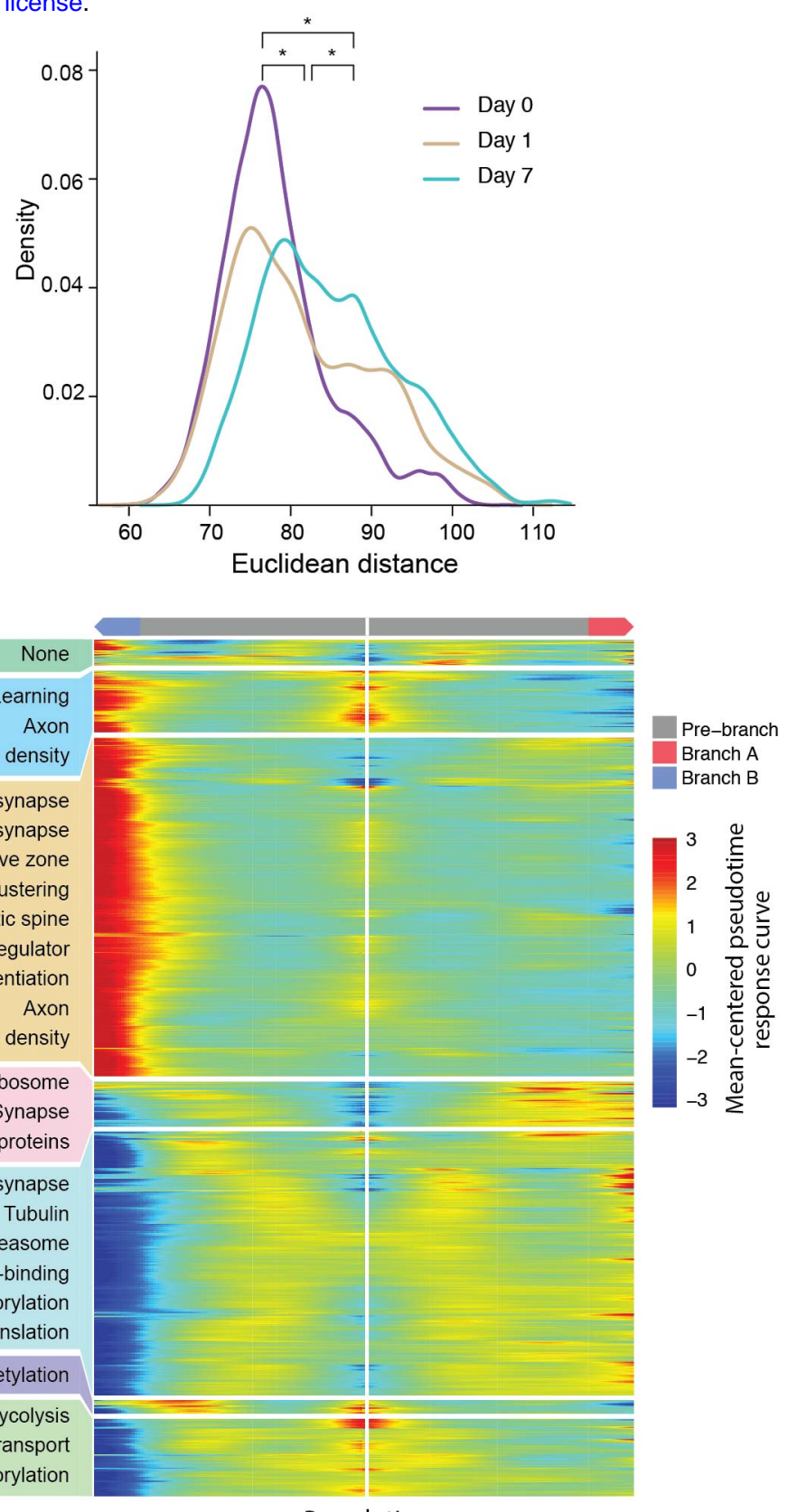

$\leftarrow$ Pseudotime $\rightarrow$

Presynaptic proteins

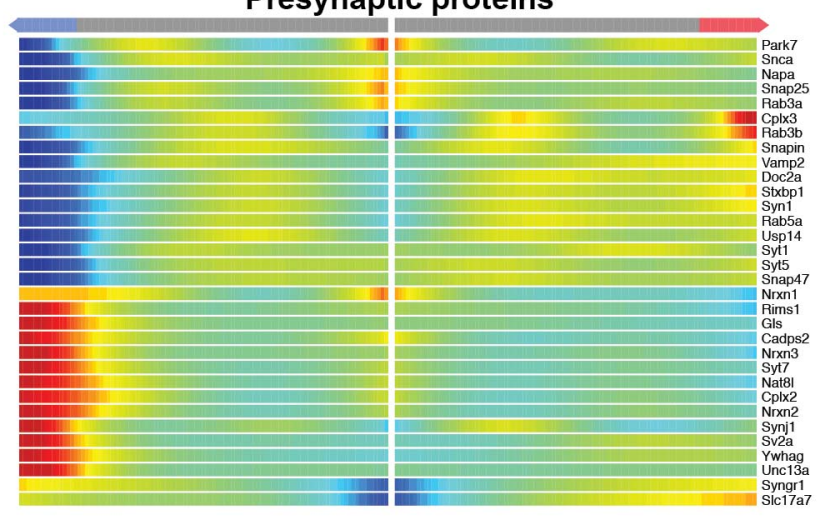

\section{Neurotransmitter receptors}

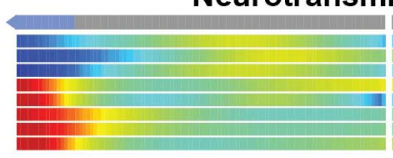

